\title{
HIGHER HOMOTOPY OPERATIONS
}

\author{
DAVID BLANC AND MARTIN MARKL
}

\begin{abstract}
We provide a general definition of higher homotopy operations, encompassing most known cases, including higher Massey and Whitehead products, and long Toda brackets. These operations are defined in terms of the $W$-construction of Boardman and Vogt, applied to the appropriate diagram category; we also show how some classical families of polyhedra (including simplices, cubes, associahedra, and permutahedra) arise in this way.
\end{abstract}

\section{INTRODUCTION}

Higher homotopy operations have a long history, starting with Toda brackets, Massey products, and Adem's secondary cohomology operations. Secondary homotopy and cohomology operations have been exploited with great effect - for example, in [As, BJM, MP, PS - but beyond attempts by Spanier (see $\$ 1.2$ below), there has been no systematic approach to higher homotopy operations in general, though they have appeared sporadically in the literature (e.g., in [Wa]), and higher cohomology operations have been studied by Maunder and others (ibid.). However, there has been a certain revival of interest in higher operations in algebraic contexts (see, for instance, [R, Ald, Tad), which may perhaps justify this attempt to set up the foundations of the subject on a new basis.

We define higher order homotopy operations as the obstruction to making a homotopycommutative diagram of spaces $\mathcal{A}: \Gamma \rightarrow$ ho $\mathcal{T}_{*}$ strictly commutative (where $\Gamma$ is a certain finite directed category which we term a "lattice"). This obstruction is defined in terms of the well-known $W$-construction of Boardman and Vogt, which takes a particularly convenient form for lattices, and yields some interesting families of polyhedra as a side benefit (see Section 1 below).

It should be emphasized that our goal here is to define the concept of a higher homotopy operation, rather than to describe such an explicit obstruction theory for rectifying diagrams, as in [DKS]. Giving an explicit dictionary for translating between the cohomology obstructions provided by Dwyer, Kan and Smith (or Spanier's approach, described below), and our description in terms of higher operations appears to be a difficult, though interesting, question.

Date: June 5, 2001; revised June 12, 2002.

Second author partially supported by grant GA AV ČR 1019203. 
This different viewpoint allows us to sweep the rather messy description of coherent vanishing of the lower operations under the carpet, giving the necessary and sufficient conditions in order that a higher order operation be defined directly in terms of an appropriate map $\mathcal{A}\left(v_{\text {init }}\right) \rtimes b \mathcal{P} \rightarrow \mathcal{A}\left(v_{\text {fin }}\right) \quad$ (see $\S 3.4$ below).

It should be observed that the theory we describe here is still somewhat ad hoc; our goal is to present a uniform treatment of the main known examples, without trying to obtain the most general definition possible. Philosophically, higher homotopy operations are connected with higher homotopies, which arise when one tries to lift a commutative diagram in the homotopy category to topological spaces (they are thus related in principle to operads, although it seems that the relation has never been made explicit). Therefore, a more general definition would perhaps require a satisfactory homotopy theory of $n$-categories, which is not yet available.

Note that, in practice, higher operations often appear in an algebraic form, as differentials in spectral sequences (for example, [As, Ch. 2], or [B2, Prop. 4.2.5]), as Ext classes (cf. Mar, Ch. 16, 3]), and so on; these often serve as an efficient means of computing such operations (e.g., [B1, §6]). But one should think of the operation itself as the intrinsic homotopy-theoretic fact, which may manifest itself in different (seemingly unrelated) algebraic guises.

1.1. Notation. The category of compactly generated topological spaces is denoted by $\mathcal{T}$, and that of pointed connected compactly generated spaces by $\mathcal{T}_{*}$. Their homotopy categories are denoted by ho $\mathcal{T}$ and ho $\mathcal{T}_{*}$ respectively. The category of simplical sets will be denoted by $\mathcal{S}$.

Let $\mathbb{N}_{+}$denote the category of finite sets $[\boldsymbol{n}]:=\{0,1,2, \ldots, n\} \quad(n=-1,0,1, \ldots$, where $[-1]:=\emptyset)$, with order-preserving monomorphisms as maps. The morphisms are generated by the inclusions $d_{n}^{i}:[\boldsymbol{n}]=\{0,1,2, \ldots, n\} \cong\{0,1, \ldots, \hat{i}, \ldots, n+1\} \hookrightarrow[\boldsymbol{n}+\mathbf{1}]$ $(i=0,1, \ldots, n+1)$. We denote by $\mathbb{N}$ the full subcategory of non-empty finite sets in $\mathbb{N}_{+}$(i.e., omit $[-1]$ ). A functor $T: \mathbb{N}^{o p} \rightarrow \mathcal{C}$ is called a $\Delta$-simplicial (or: restricted simplicial) object over $\mathcal{C}$; this is just a simplicial object without degeneracies.

1.2. Other approaches to higher operations. Toda's definition of what we now call Toda brackets in [T1] (see [T2, Ch. I] and Example 3.12 below) was the first example of a secondary homotopy operation stricto sensu, although Adem's secondary cohomology operations (see $\mathrm{Am}$ ), and Massey's triple products in cohomology (see $\mathbb{M}$ ] and Sec. 5 below) appeared at about the same time.

In all three cases there was no attempt to provide a theoretical framework for such operations; it was Adams, in [As, Ch. 3], who first produced a general definition of secondary cohomology operations, based on "universal examples" - cohomology classes in the fiber of a map between mod $p$ generalized Eilenberg-Mac Lane objects (GEMs) and explained how the so-called stable secondary operations correspond to relations in 
the Steenrod algebra. This approach was generalized by Maunder in his thesis to $n$-th order cohomology operations, using chain complexes (or towers) of length $n$; he also gave necessary and sufficient conditions in order for a higher-order operation to be defined, in terms of certain "pyramids" of lower order operations (see [Mau]).

Later, Holtzman (in [ [H]) described an alternative version of $n$-th order cohomology operations based on the $p$-divisibility of certain "pseudo primary operations." Finally, Klaus (in [K]) defines unstable higher order operations in terms of (not necessarily linear) natural transformations on cochains - generalizing the approach of Kristensen in $[\mathrm{Kn}, \mathrm{KK}]$.

However, in all these approaches we do not see the combinatorial underpinning (i.e., the lattice $\Gamma$ ) of our more general definition, essentially because the homotopy category of cochain complexes (or of GEMs) is additive.

In [Sp2], Spanier gave a general theory of higher order operations (extending the definition of secondary operations given in $[\mathbf{S p} 1]$, somewhat similar in spirit to the approach we propose here: an $(n+1)$-st order operation is defined as a set of cohomology classes $\mathcal{O}^{n} \subset H^{n}\left(K ; \Gamma_{n}\right)$, where $K$ is a simplicial complex (corresponding essentially to our $b \mathcal{P}$ - see $\$ 2.13$ below), and the coefficients $\Gamma_{n}$ are a stack ("cosheaf of groups") on $K$, defined

$$
\Gamma_{n}(\sigma):=\pi_{n} \Phi(\sigma) \quad \text { for } \quad \sigma \in K,
$$

where $\Phi(\sigma)$ is the topological space assigned to the simplex $\sigma \in K$ by a given carrier ("cosheaf of spaces"), and $\pi_{n}$ is as usual its $n$-th homotopy group.

However, Spanier does not explain how $\Phi$ and $K$ may be constructed from a diagram $\mathcal{A}: \Gamma \rightarrow$ ho $\mathcal{T}_{*}$, so the interpretation of the higher operations as obstructions to rectification is obscured.

1.3. Acknowledgements. We would like to thank Rainer Vogt for explaining some of the secrets of [BV] to the second author. The first author would like to thank the Department of Mathematics at Northwestern University for their hospitality during the period when this paper was completed. Thanks are also due to the referee for his helpful comments and suggestions.

\section{LAtTiCES And THE $W$-CONSTRUCTION}

Motivated by the examples of [B3, B4], in Section 3] we are going to define higher homotopy operations for pointed topological spaces. This section contains some auxiliary material for definitions in Section 3 . 
2.1. Definition. For the purposes of this paper, a lattice will be a finite directed category $\Gamma$ with weak initial object $v_{\text {init }}=v_{\text {init }}(\Gamma)$ and weak terminal object $v_{\text {fin }}=v_{\text {fin }}(\Gamma)$. This means simply that for every object (or node) $w$ of $\Gamma$, there is at least one map (or arrow) from $v_{\text {init }}$ to $w$, and at least one from $w$ to $v_{\text {fin }}$ - and that moreover there is a unique maximal arrow $\phi_{\max }: v_{\text {init }} \rightarrow v_{\text {fin }}$. We will always assume that $\Gamma$ is non-trivial - that is, there exists a node $w$ of $\Gamma$ with $v_{\text {init }} \neq w \neq v_{\text {fin }}$.

We shall denote the finite set of objects (or nodes) of $\Gamma$ by $V$. A composable sequence of $k$ arrows in $\Gamma, \Phi=\left\langle v_{k} \stackrel{f_{k}}{\rightarrow} v_{k-1} \rightarrow \cdots \rightarrow v_{1} \stackrel{f_{1}}{\rightarrow} v_{0}\right\rangle$, will be called a $k$-chain. We sometimes denote it more briefly by $f_{1} f_{2} \cdots f_{k}$ (note the reversed order). If it starts at $v_{\text {init }}$ and ends at $v_{\text {fin }}$, we say $\Phi$ is a maximal chain.

2.2. Example. For each $n \geq 1$ we denote by $L_{n}$ the lattice generated by $n$ composable morphisms

$$
v_{\text {init }} \stackrel{f_{1}}{\longrightarrow} v_{1} \stackrel{f_{2}}{\longrightarrow} v_{2} \cdots v_{n-1} \stackrel{f_{n}}{\longrightarrow} v_{\text {fin }}
$$

(and no further relations).

2.3. Remark. Because our category $\Gamma$ is directed, and thus has no non-trivial loops, we shall treat it as a non-unital category - i.e., we omit the identity morphisms, so $\operatorname{Hom}_{\Gamma}(v, v)=\emptyset$, and we can think of $\Gamma$ simply as a directed graph with commutation relations (which are completely determined by a set of distinguished "commutative triangles" in $\Gamma$, with sides $f, g$, and $f \circ g$ ).

2.4. The $W$-construction. A lattice $\Gamma$, being a special kind of a category, is in particu-

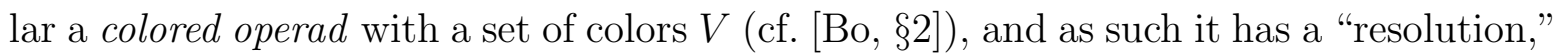
denoted by $W \Gamma$ and called the bar construction on $\Gamma$ by Boardman and Vogt (cf. $\mathbb{B} \nabla$, III, §1]). We would like to think of it as a cofibrant replacement in a hypothetical model category structure on the category of all (small) categories.

2.5. Definition. Let $\Gamma_{n+1}(u, v)$ be the discrete space of all $(n+1)$-chains

$$
\left\langle u=v_{n+1} \stackrel{f_{n+1}}{\longrightarrow} v_{n} \rightarrow \cdots \rightarrow v_{1} \stackrel{f_{1}}{\rightarrow} v_{0}=v\right\rangle .
$$

$I:=[0,1]$ denotes as usual the unit interval.

For $u, v \in V$, let

$$
W \Gamma(u, v):=\bigsqcup_{n \geq 0} \Gamma_{n+1}(u, v) \times I^{n} / \sim
$$

with the relation $\sim$ defined as follows: if

$$
\begin{array}{lllllllllll}
f_{1} & \circ_{t_{1}} & f_{2} & \circ_{t_{2}} & \cdots & \circ_{t_{n-1}} & f_{n} & \circ_{t_{n}} & f_{n+1}
\end{array}
$$

denotes the point

$$
\left\langle u \stackrel{f_{n+1}}{\longrightarrow} v_{n} \rightarrow \cdots \rightarrow v_{1} \stackrel{f_{1}}{\rightarrow} v\right\rangle \times\left(t_{n}, \ldots, t_{1}\right) \in \Gamma_{n+1}(u, v) \times I^{n},
$$


then

$$
f_{1} \circ_{t_{1}} f_{2} \circ_{t_{n-1}} \cdots \circ_{t_{n-1}} f_{n} \circ_{t_{n}} f_{n+1} \sim f_{1} \circ_{t_{1}} \cdots \circ_{t_{i-1}}\left(f_{i} f_{i+1}\right) \circ_{t_{i+1}} \cdots \circ_{t_{n}} \circ f_{n+1}
$$

if $t_{i}=0$ for some $1 \leq i \leq n$.

In (2.6), $\left(f_{i} f_{i+1}\right)$ denotes $f_{i}$ composed with $f_{i+1}$. Loosely speaking, the relation means that $\circ_{t}$ becomes, for $t=0$, an ordinary composition of morphisms in $\Gamma$. The categorial composition in $W \Gamma$ is given by the concatenation:

$$
\left(f_{1} \circ_{t_{1}} \cdots \circ_{t_{l}} f_{l+1}\right) \circ\left(g_{1} \circ_{u_{1}} \cdots \circ_{u_{k}} g_{k+1}\right):=\left(f_{1} \circ_{t_{1}} \cdots \circ_{t_{l}} f_{l+1} \circ_{1} g_{1} \circ_{u_{1}} \cdots \circ_{u_{k}} g_{k+1}\right) \text {. }
$$

Definition 2.5 is based on the definition given in [SV], p. 367]; our version is simpler because we work without units.

2.7. The cubical structure. The category $W \Gamma$ is enriched not only over topological spaces, but also cubically - with a cubical set $\operatorname{Hom}_{W \Gamma}(u, v)$ associated to each pair of objects $u, v$ of $\Gamma$ as follows:

For each $(n+1)$-chain

$$
\Phi=\left\langle u \stackrel{f_{n+1}}{\longrightarrow} v_{n} \stackrel{f_{n}}{\longrightarrow} \cdots \stackrel{f_{2}}{\longrightarrow} v_{1} \stackrel{f_{1}}{\longrightarrow} v\right\rangle
$$

from $u$ to $v$, we have an $n$-dimensional cube $C[\Phi]$ of equivalence classes of points of $\{\Phi\} \times I^{n} \subset \Gamma_{n+1}(u, v) \times I^{n}$. Clearly,

(a) the $k$-th 0 -facet of $C[\Phi] \quad(1 \leq k \leq n)$ equals to $C\left[\Phi^{(k)}\right]$, where $\Phi^{(k)}$ is obtained from $\Phi$ by composing the maps at the $k$-th internal node of $\Phi$;

(b) the $k$-th 1 -facet of $C[\Phi]$ equals to the product of the two cubes $C\left[\Phi_{k}^{\prime}\right]$ and $C\left[\Phi_{k+1}^{\prime \prime}\right]$ of dimensions $k-1$ and $n-k$ respectively, where

$$
\Phi_{k}^{\prime}:=\left\langle v_{k} \stackrel{f_{k}}{\longrightarrow} v_{k-1} \cdots v_{1} \stackrel{f_{1}}{\rightarrow} v\right\rangle
$$

and

$$
\Phi_{k}^{\prime \prime}:=\left\langle u \stackrel{f_{n+1}}{\longrightarrow} v_{n} \cdots v_{k+1} \stackrel{f_{k+1}}{\longrightarrow} v_{k}\right\rangle .
$$

Each facet of the cube $C[\Phi]$ has the form $\left\{\begin{array}{llllll}f_{1} & \circ_{t_{1}} & f_{2} & \cdots & \circ_{t_{n}} & f_{n+1}\end{array} \mid t_{i} \in\{0,1\}\right\}$ for some fixed $1 \leq i \leq n$, and a $k$-dimensional face of $C[\Phi]$ is defined by requiring $(n-k)$ of the parameters $\left(t_{1}, \ldots, t_{n}\right)$ to take a fixed value in $\{0,1\}$.

In particular, vertices of $C[\Phi]$ are points for which all $t_{i} \in\{0,1\}$. Using relation (2.6), we can remove from $f_{1} \circ_{t_{1}} f_{2} \cdots \circ_{t_{n}} f_{n+1}$ all operations $\circ_{0}$ by composing the adjacent morphisms. Therefore the vertices of the cubes of $W \Gamma$ are in fact indexed by chains of composable morphisms. For example, the vertex $f_{1} \circ_{1} f_{2} \circ_{0} f_{3} \circ_{1} f_{4}$ of $C[\Phi]$ with

$$
\Phi=\left\langle u \stackrel{f_{4}}{\longrightarrow} v_{3} \stackrel{f_{3}}{\longrightarrow} v_{2} \stackrel{f_{2}}{\longrightarrow} v_{1} \stackrel{f_{1}}{\longrightarrow} v\right\rangle
$$

is indexed by the 3 -chain $f_{1}\left(f_{2} f_{3}\right) f_{4}$, vertex $f_{1} \circ_{0} f_{2} \circ_{0} f_{3} \circ_{1} f_{4}$ by the 2 -chain $\left(f_{1} f_{2} f_{3}\right) f_{4}$, and so on. 
The (cubical) k-skeleton of $W \Gamma$, denoted by $\mathrm{sk}_{k} W \Gamma$, is the subcategory generated by the union of all faces of dimension $\leq k$.

There is an obvious augmentation functor $\varepsilon: W \Gamma \rightarrow \Gamma$, with a "section" $\sigma: \Gamma \rightarrow$ $W \Gamma$, which actually lands in the 0 -skeleton $\mathrm{sk}_{0} W \Gamma$. The fiber $\varepsilon^{-1}(f)$ is contractible for each map $f$ of $\Gamma$.

2.8. Remark. We will be interested mainly in the single cubical set $\operatorname{Hom}_{W \Gamma}\left(v_{\text {init }}, v_{\text {fin }}\right)$, which will be called the total mapping space of $W \Gamma$, and denoted by $\mathcal{P}_{\Gamma}$, or simply $\mathcal{P}$. However, we do need the full structure of $W \Gamma$ as a cubically enriched category and this is expressed by relations among the various subcomplexes of $\mathcal{P}$.

Let us abbreviate the notation of Definition 2.5 by writing $f g$ for the composition $f \circ_{0} g,(f)(g)$ for the composable sequence $f \circ_{1} g$, and $f \circ g$ for the 1-cube $f \circ_{t} g$ $(0 \leq t \leq 1)-$ and similarly in higher dimensions.

2.9. Example. For the lattice $L_{3} \quad(\S 2.2)$ with three composable morphisms $v_{\text {init }} \stackrel{h}{\rightarrow}$ $v_{3} \stackrel{g}{\rightarrow} v_{2} \stackrel{f}{\rightarrow} v_{\text {fin }}$, we see that $W L_{3}\left(v_{\text {init }}, v_{\text {fin }}\right)$ is the square depicted in Figure 2.10, where the arrows, indicating the direction $0 \rightarrow 1$, are included to identify more easily the two faces of each sub-cube.

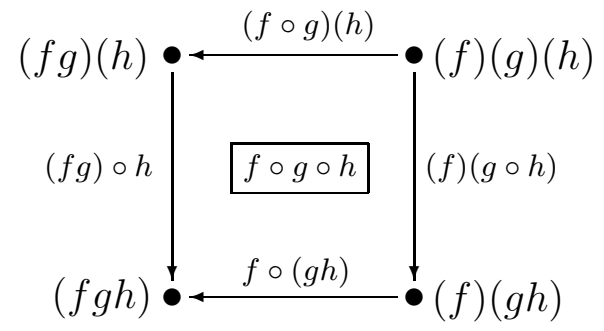

Figure 2.10. The square $W L_{3}\left(v_{\text {init }}, v_{\text {fin }}\right)$

2.11. Example. Similarly, for the lattice $L_{4}$ with four composable morphisms $v_{\text {init }} \stackrel{k}{\rightarrow}$ $v_{4} \stackrel{h}{\rightarrow} v_{3} \stackrel{g}{\rightarrow} v_{2} \stackrel{f}{\rightarrow} v_{\text {fin }}$, we find that $W L_{4}\left(v_{\text {init }}, v_{\text {fin }}\right)$ is the cube in Figure 2.12 .

2.13. Definition. The category $b W \Gamma$ is defined to be the cubical subcategory of $W \Gamma$ with $\operatorname{Obj} b W \Gamma=\operatorname{Obj} W \Gamma=\operatorname{Obj} \Gamma$, and with morphisms given by $b W \Gamma(u, v):=$ $W \Gamma(u, v)$ if $(u, v) \neq\left(v_{\text {init }}, v_{\text {fin }}\right)$, while

$$
b W \Gamma\left(v_{\text {init }}, v_{\text {fin }}\right):=\bigcup\left\{\alpha \circ \beta \mid \beta \in W \Gamma\left(v_{\text {init }}, w\right), \alpha \in W \Gamma\left(w, v_{\text {fin }}\right), v_{\text {init }} \neq w \neq v_{\text {fin }}\right\} .
$$

As usual, we abbreviate $\operatorname{Hom}_{b W \Gamma}(u, v)$ to $b W \Gamma(u, v)$ and $\operatorname{Hom}_{W \Gamma}(u, v)$ to $W \Gamma(u, v)$.

Thus $b W \Gamma\left(v_{\text {init }}, v_{\text {fin }}\right)$ consists of all decomposable morphisms, so that as a cubical set its facets are of the form $C_{k}^{\prime}[\Phi]:=\left\{\left(t_{1}, \ldots, t_{n}\right) \in C[\Phi]: t_{k}=1\right\}$ for some fixed $1 \leq k \leq n$ and maximal chain $\Phi$. We will sometimes call these the basic facets 


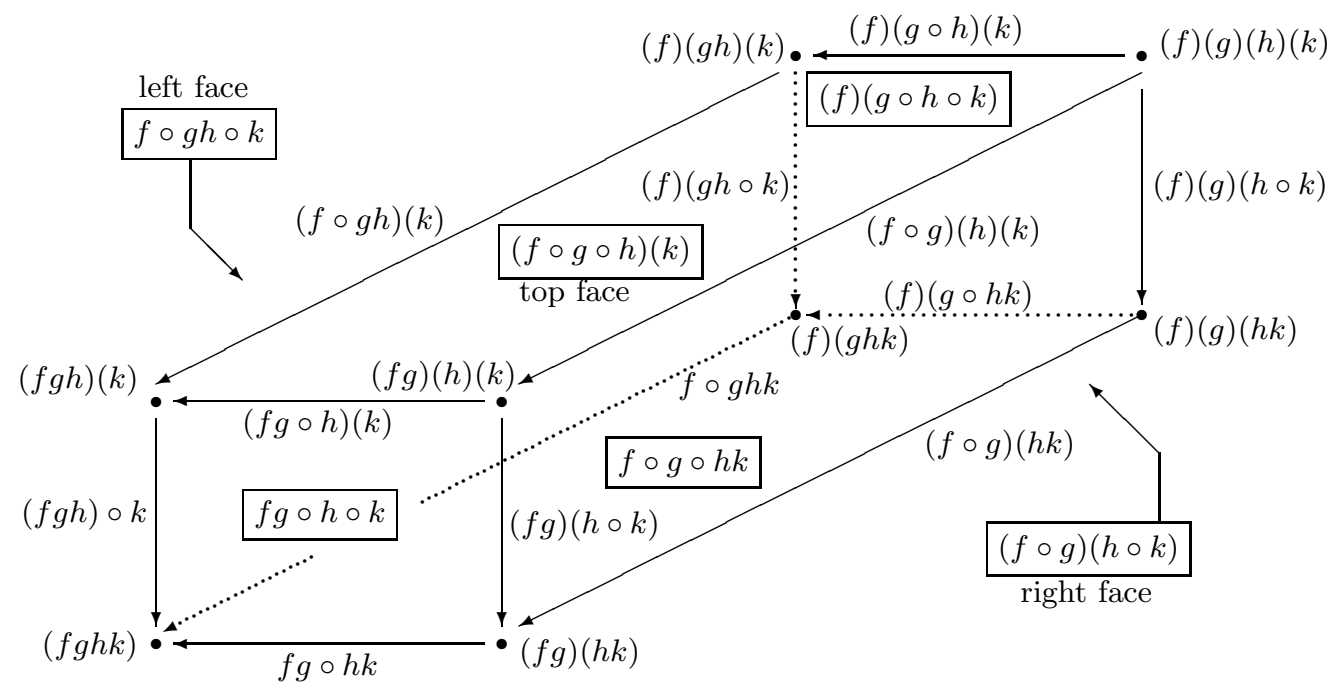

Figure 2.12. The cube $\mathcal{P}=W L_{4}\left(v_{\text {init }}, v_{\text {fin }}\right)$

of $W \Gamma\left(v_{\text {init }}, v_{\text {fin }}\right)$, and $b W \Gamma\left(v_{\text {init }}, v_{\text {fin }}\right)$ will be called the basis of $W \Gamma$. We denote $b W \Gamma\left(v_{\text {init }}, v_{\text {fin }}\right)$ by $b \mathcal{P}$ and consider it as a (cubical) subspace of $\mathcal{P}$. For instance, in Example 2.9 above, the basis of $W L_{3}$ consists of the top and right edges of the square in Figure 2.10.

2.14. Remark. The 0 -skeleton $\mathrm{sk}_{0} W \Gamma$ is the free nonunital category on the set of arrows of $\Gamma$. Therefore each arrow $f \in \Gamma$ is replicated in $W \Gamma$, but the inclusion $\sigma: \Gamma \rightarrow W \Gamma$ defined in this way is not a functor. So for $u, v \in V$, we will sometimes identify elements $f \in \Gamma(u, v)$ with their images $\sigma(f) \in W \Gamma(u, v)$, that is, consider $\Gamma(u, v)$ as a subset of $W \Gamma(u, v)$, and emphasize this by writing $\Gamma \subset \mathrm{sk}_{0} W \Gamma$.

Similarly, $\operatorname{sk}_{0} b W \Gamma$ is the free nonunital category on the set of arrows of $\Gamma \backslash\left\{\phi_{\max }\right\}$.

2.15. Proposition. For any lattice $\Gamma, \mathcal{P}=W \Gamma\left(v_{\text {init }}, v_{\mathrm{fin}}\right)$ is combinatorially isomorphic to the cone $C b \mathcal{P}$ on its basis $b \mathcal{P}:=b W \Gamma\left(v_{\text {init }}, v_{\text {fin }}\right)$, with vertex of the cone corresponding to the unique maximal 1-chain $\left\langle v_{\mathrm{init}} \stackrel{\phi_{\max }}{\longrightarrow} v_{\mathrm{fin}}\right\rangle$.

Proof. Recall that $C b \mathcal{P}$ is the quotient $(I \times b \mathcal{P}) /(\{0\} \times b \mathcal{P})$. Define a continuous map $\alpha: C b \mathcal{P} \rightarrow \mathcal{P}$ as follows.

Let $s \times\left[f_{1} \circ_{t_{1}} f_{2} \cdots f_{n} \circ_{t_{n}} f_{n+1}\right]$ be a point of $I \times b \mathcal{P}$, where

$$
\left\langle v_{\text {init }} \stackrel{f_{n+1}}{\longrightarrow} v_{n} \stackrel{f_{n}}{\longrightarrow} \cdots \stackrel{f_{2}}{\longrightarrow} v_{1} \stackrel{f_{1}}{\longrightarrow} v_{\text {fin }}\right\rangle
$$

is a maximal chain, $s, t_{1}, \ldots, t_{n} \in I$, and $[-]$ denotes the equivalence class, as usual. Let

$$
\tilde{\alpha}\left(s \times\left[f_{1} \circ_{t_{1}} f_{2} \cdots f_{n} \circ_{t_{n}} f_{n+1}\right]\right):=\left[f_{1} \circ_{s t_{1}} f_{2} \cdots f_{n} \circ_{s t_{n}} f_{n+1}\right] \in \mathcal{P} .
$$


If $s=0$, clearly

$$
\tilde{\alpha}\left(s \times\left[f_{1} \circ_{t_{1}} f_{2} \cdots f_{n} \circ_{t_{n}} f_{n+1}\right]\right)=\left[f_{1} \circ_{0} f_{2} \cdots f_{n} \circ_{0} f_{n+1}\right]=\left[\phi_{\max }\right] .
$$

Thus $\tilde{\alpha}(\{0\} \times b \mathcal{P})=\left\{\phi_{\max }\right\}$, so that $\tilde{\alpha}$ induces a map $\alpha: C b \mathcal{P} \rightarrow \mathcal{P}$ which sends the vertex of the cone to $\left[\phi_{\max }\right] \in \mathcal{P}$.

The inverse of $\alpha$ can be constructed as follows: let $f_{1} \circ_{t_{1}} f_{2} \cdots f_{n} \circ_{t_{n}} f_{n+1}$ represent a point of $\mathcal{P}$, and let $s:=\max \left\{t_{1}, \ldots, t_{n}\right\}$. If $s=0$ (which means that $t_{i}=0$ for all $i)$, let $\beta\left(\left[f_{1} \circ_{t_{1}} f_{2} \cdots f_{n} \circ_{t_{n}} f_{n+1}\right]\right)$ equal to the vertex of $C b \mathcal{P}$. If $s>0$, then

$$
\beta\left(f_{1} \circ_{t_{1}} f_{2} \cdots f_{n} \circ_{t_{n}} f_{n+1}\right):=\pi\left(s \times\left[f_{1} \circ_{t_{1} / s} f_{2} \cdots f_{n} \circ_{t_{n} / s} f_{n+1}\right]\right) \in C b \mathcal{P},
$$

where $\pi: I \times b \mathcal{P} \rightarrow C b \mathcal{P}$ is the projection. Observe that at least one of $t_{1} / s, \ldots, t_{n} / s$ equals 1 , so indeed $\left[f_{1} \circ_{t_{1} / s} f_{2} \cdots f_{n} \circ_{t_{n} / s} f_{n+1}\right] \in b \mathcal{P}$.

It is easily verified that the map $\beta: \mathcal{P} \rightarrow C b \mathcal{P}$ is well-defined, and inverse to $\alpha$.

2.16. Example. In Example 2.11 above, the basis of $W L_{4}$ is the union of the three 2-dimensional faces opposite the vertex $(f g h k)$, as depicted in Figure 2.17; and the cube of Figure 2.12 is indeed homeomorphic to the cone on $b W L_{4}$.

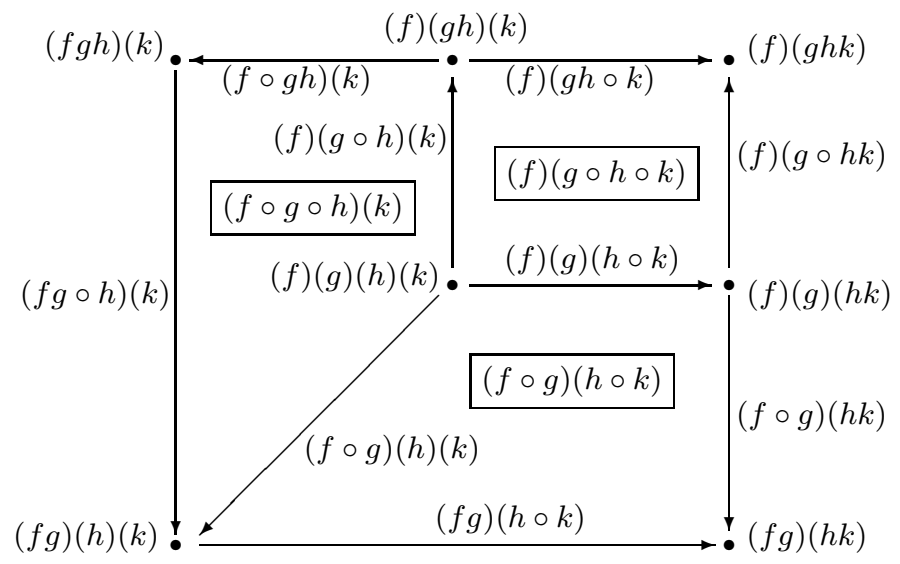

Figure 2.17. Basis for $W L_{4}\left(v_{\text {init }}, v_{\text {fin }}\right)$

We now list some properties of the $W$-construction $W \Gamma$ which will be needed in Section 3, using the following terminology:

For a finite set $V$ (which will be the set of objects of the category $\Gamma$ ), a $V$-graded space will be a sequence $\mathbf{X}=\{X(v) \mid v \in V\}$ of well-pointed topological spaces; a homotopy equivalence between $V$-graded spaces $\mathbf{X}$ and $\mathbf{Y}$ is a sequence $\mathbf{h}=\left\{h_{v}\right.$ : $X(v) \rightarrow Y(v) \mid v \in V\}$ of homotopy equivalences in $\mathcal{T}_{*}$; if such an $\mathbf{h}$ exists, we say $\mathbf{X}$ and $\mathbf{Y}$ have the same homotopy type.

If $\Xi$ is a (topological) category with $\operatorname{Obj}(\Xi)=V$, a $V$-graded space $\mathbf{X}$ is called a $\Xi$-space if there is a $\Xi$-structure on $\mathbf{X}$ - that is, a (continuous) functor $G: \Xi \rightarrow \mathcal{T}_{*}$ such that $G(v)=X(v)$ for all $v \in V$. 
The following statement, which we will need for the proof of Theorem 3.8, is a simplified version of [BV, Theorem IV.4.37] which was formulated there for an arbitrary colored operad. The proof makes use of units, but applies by a slight modification in our case too.

2.18. Theorem. A V-graded space $\mathbf{X}$ admits a $W \Gamma$-structure if and only if it is homotopy equivalent to a $\Gamma$-space. More precisely,

(a) suppose that $\mathbf{X}$ is a $W \Gamma$-space with the structure given by a functor $\mathcal{B}: W \Gamma \rightarrow \mathcal{T}_{*}$. Then there exists a $\Gamma$-space $\mathbf{Y}$ given by a functor $F: \Gamma \rightarrow \mathcal{T}_{*}$, and a homotopy equivalence $\mathbf{h}: \mathbf{X} \stackrel{\sim}{\rightarrow} \mathbf{Y}$ such that the diagram

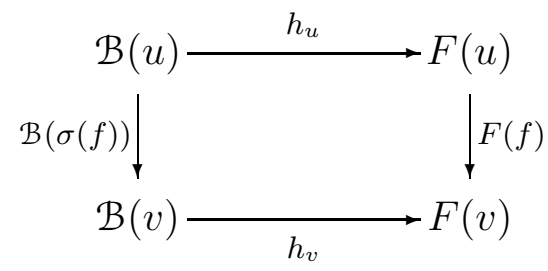

is homotopy commutative for all arrows $f: u \rightarrow v$ of $\Gamma$.

(b) Conversely, if $\mathbf{Y}$ is a $\Gamma$-space, with structure given by $F: \Gamma \rightarrow \mathcal{T}_{*}$, and $\mathbf{h}: \mathbf{X} \rightarrow$ $\mathbf{Y}$ is a homotopy equivalence of $V$-graded spaces, then there is a W $\Gamma$-structure $\mathcal{B}: W \Gamma \rightarrow \mathcal{T}_{*}$ on $\mathbf{X}$ such that the diagram (2.19) is homotopy commutative for all $f: u \rightarrow v$ as above.

The strict $\Gamma$-space $\mathbf{Y}$ whose existence is claimed in the first part of the Theorem is called a rectification of the $W \Gamma$-space $\mathbf{X}$. The original result in [BV], Thm. IV.4.37] in fact states that the homotopy equivalence $\mathbf{h}$ can be equipped with a structure of a strongly homotopy $\Gamma$-map, (in an appropriate sense). The homotopy commutativity of (2.19) then follows from the existence of this structure.

For a subcategory $U$ of $W \Gamma, u, v \in V$, and $n \geq 0$, let $U_{n+1}(u, v) \subset \Gamma_{n+1}(u, v) \times I^{n}$ denote the set of all elements representing morphisms in $U$. Following [SV], we call $U$ admissible if each morphism of $U$ that decomposes in $W \Gamma$, decomposes also in $U$, and if, moreover, the inclusion

$$
U_{n+1}(u, v) \cup \Gamma_{n+1}(u, v) \times \partial I^{n} \hookrightarrow \Gamma_{n+1}(u, v) \times I^{n}
$$

is a closed cofibration for all $n, u, v$. Observe that both $\operatorname{sk}_{0} W \Gamma$ and $\operatorname{sk}_{0} b W \Gamma$ are admissible subcategories of $W \Gamma$; in both cases $U_{n+1}(u, v) \subset \Gamma_{n+1}(u, v) \times \partial I^{n}$.

The above condition is a bit weaker than the one in [SV, p. 374], because we do not have units. The following statement is a version of [SV, Prop. 4.4], which will be used in the proof of Proposition 3.17:

2.20. Proposition. Let $U$ be an admissible subcategory of $W \Gamma$. Suppose we are given a functor $F_{0}: W \Gamma \rightarrow \mathcal{T}_{*}$ and a homotopy through functors $H_{t}: U \rightarrow \mathcal{T}_{*}$ such that $H_{0}=\left.F_{0}\right|_{U}$. Then there exists an $F_{t}$ extending both $F_{0}$ and $H_{t}$. 
2.21. Remark. There is a version of $W \Gamma$, based on a construction of Cordier and Porter (in [CP, §2]), which is enriched over simplicial, rather than cubical, sets:

Given a lattice $\Gamma$ as above, the category $W_{s} \Gamma$ has the same objects as $\Gamma$, and for each pair of nodes $(u, v)$ of $\Gamma, W_{s} \Gamma(u, v) \in \mathcal{S}$ is a simplicial set with one $r$-simplex $\sigma_{(\mathcal{U}, \Phi)}$

for each chain $\Phi=\left\langle u=v_{n+1} \stackrel{f_{n+1}}{\longrightarrow} v_{n} \stackrel{f_{n}}{\longrightarrow} \cdots \stackrel{f_{2}}{\longrightarrow} v_{1} \stackrel{f_{1}}{\longrightarrow} v_{0}=v\right\rangle=f_{1} f_{2} \cdots f_{n} f_{n+1}$ and each partition $\mathcal{U}=\left(U_{1}, \ldots, U_{r}\right)$ of a subset $U \subset\left\{v_{1}, \ldots, v_{n}\right\}$ of the set of internal nodes of $\Phi$, with each of the sets $U_{i}$ nonempty. The faces of $\sigma$ are defined by:

(i) $d_{0}(\sigma):=\sigma_{\left(\mathcal{U}^{(0)}, \Phi^{\prime}\right)}$, where $\Phi^{\prime}$ is obtained from $\Phi$ by carrying out the compositions at each node $v_{i} \in U_{1}$, and $\mathcal{U}^{(0)}:=\left(U_{2}, \ldots, U_{r}\right)$.

(ii) $d_{j}(\sigma):=\sigma_{\left(\mathcal{U}^{(j)}, \Phi\right)}$, where $\mathcal{U}^{(j)}:=\left(U_{1}, \ldots, U_{j-1}, U_{j} \cup U_{j+1}, U_{j+2}, \ldots, U_{r}\right)$ for $0<$ $j<r$.

(iii) $d_{r}(\sigma):=\sigma_{\left(\mathcal{U}^{(r)}, \Phi\right)}$, where $\mathcal{U}^{(r)}:=\left(U_{1}, \ldots, U_{r-1}\right)$.

The degenerate simplices are obtained by allowing partitions with $U_{j}=\emptyset$, and the simplicial composition map is defined by concatentation of chains in the obvious way. Note that $W_{s} \Gamma(u, v)$ (or the corresponding simplicial complex) clearly provides a canonical triangulation of the cubical set $W_{s} \Gamma(u, v)$.

The construction $W_{s} \Gamma$ can be thought of as a functorial bar resolution:

$$
B\left(\mathrm{sk}_{0} W, \mathrm{sk}_{0} W_{0}, \Gamma\right)
$$

(cf. [May, §9]).

\section{Higher homotopy operations}

With the constructions of Section 2 at hand, we are now in a position to define our basic object of interest:

3.1. Definition. Initial data for a higher homotopy operation is a lattice $\Gamma$, together with a $\Gamma$-diagram up-to-homotopy - i.e., a functor $\mathcal{A}: \Gamma \rightarrow$ ho $\mathcal{T}_{*}$.

Note that if $\sigma: \Gamma \rightarrow W \Gamma$ is the (non-functorial) canonical section of the augmentation $\varepsilon: W \Gamma \rightarrow \Gamma$, then for every continuous functor $F: W \Gamma \rightarrow \mathcal{T}_{*}$, the composite $\pi \circ F \circ \sigma:$ $\Gamma \rightarrow$ ho $\mathcal{T}_{*}$ is a functor, and $\pi \circ F$ factors through $\varepsilon$, where $\pi: \mathcal{T}_{*} \rightarrow$ ho $\mathcal{T}_{*}$ is the obvious projection functor.

A rectification of the initial data $\mathcal{A}: \Gamma \rightarrow$ ho $\mathcal{T}_{*}$ is then a strict $\Gamma$-diagram realizing $\mathcal{A}$ - i.e., a functor $F: \Gamma \rightarrow \mathcal{T}_{*}$ such that $\pi \circ F$ is naturally isomorphic to $\mathcal{A}$.

Recall that the (right) half-smash $X \rtimes K$ of topological spaces $X$ and $K$, where $X$ is pointed, is defined to be $(X \times K) /(\{*\} \times K)=X \wedge K_{+}$, where $K_{+}$is $K$ with a disjoint basepoint added. $X \rtimes K$ is again a pointed space, with the class of $\{*\} \times K$ as the distinguished point. 
Given two pointed spaces $X, Y \in \mathcal{T}_{*}$ and any space $K \in \mathcal{T}$, the following adjointness relation holds:

$$
\mathcal{T}_{*}(X \rtimes K, Y) \cong \mathcal{T}_{*}\left(X \wedge K_{+}, Y\right) \cong \mathcal{T}_{*}\left(K_{+}, \mathcal{T}_{*}(X, Y)\right) \cong \mathcal{T}\left(K, \mathcal{T}_{*}(X, Y)\right),
$$

provided one works in the category of (not necessarily connected) pointed compactlygenerated spaces.

3.3. Convention. In (3.2), we will typically have $X=\mathcal{A}\left(v_{\text {init }}\right), \quad Y=\mathcal{A}\left(v_{\text {fin }}\right)$ and $K=b \mathcal{P}$. In this case, we will make no distinction between maps $\mathcal{A}\left(v_{\text {init }}\right) \rtimes b \mathcal{P} \rightarrow \mathcal{A}\left(v_{\text {fin }}\right)$ and maps $b \mathcal{P} \rightarrow \mathcal{T}_{*}\left(\mathcal{A}\left(v_{\text {init }}\right), \mathcal{A}\left(v_{\text {fin }}\right)\right)$.

3.4. Definition. Given initial data $\mathcal{A}: \Gamma \rightarrow$ ho $\mathcal{T}_{*}$, complete data for the corresponding higher homotopy operation consists of a continuous functor $C \mathcal{A}: b W \Gamma \rightarrow \mathcal{T}_{*}$ such that $\pi \circ C \mathcal{A}=\mathcal{A} \circ\left(\left.\varepsilon\right|_{b W \Gamma}\right)$.

The corresponding higher order homotopy operation is the subset

$$
\langle\langle\mathcal{A}\rangle\rangle \subset\left[\mathcal{A}\left(v_{\text {init }}\right) \rtimes b \mathcal{P}, \mathcal{A}\left(v_{\text {fin }}\right)\right]_{\text {ho } \mathcal{T}_{*}}
$$

consisting of the homotopy equivalence classes of maps

$$
\left.C \mathcal{A}\right|_{b W \Gamma\left(v_{\text {init }}, v_{\text {fin }}\right)}: b W \Gamma\left(v_{\text {init }}, v_{\text {fin }}\right)=b \mathcal{P} \longrightarrow \mathcal{T}_{*}\left(\mathcal{A}\left(v_{\text {init }}\right), \mathcal{A}\left(v_{\text {fin }}\right)\right)
$$

induced by all possible complete data $C \mathcal{A}$ for $\mathcal{A}$.

3.5. Definition. The higher operation $\langle\langle\mathcal{A}\rangle\rangle$ is said to vanish if it contains the homotopy class of a constant map $b \mathcal{P} \longrightarrow \mathcal{T}_{*}\left(\mathcal{A}\left(v_{\text {init }}\right), \mathcal{A}\left(v_{\text {fin }}\right)\right)$.

The proof of the following statement is based on Proposition 2.15.

3.6. Proposition. The operation $\langle\langle\mathcal{A}\rangle\rangle$ vanishes if and only if there exists a continuous functor $\mathcal{B}: W \Gamma \rightarrow \mathcal{T}_{*}$ such that

$$
\pi \circ \mathcal{B}=\mathcal{A} \circ \varepsilon
$$

Proof. The vanishing of $\langle\langle\mathcal{A}\rangle\rangle$ means, by definition, that there are complete data $C \mathcal{A}$ : $b W \Gamma \rightarrow \mathcal{T}_{*}$ such that $\left.C \mathcal{A}\right|_{b W \Gamma\left(v_{\text {init }}, v_{\text {fin }}\right)}$ is homotopic to a constant map. We then define $\mathcal{B}: W \Gamma \rightarrow \mathcal{T}_{*}$ on objects by $\mathcal{B}(v):=\mathcal{A}(v)$, for each $v \in V$, and on morphisms as follows.

Recall that, for $(u, v) \neq\left(v_{\text {init }}, v_{\text {fin }}\right)$ we have $W \Gamma(u, v)=b W \Gamma(u, v)$, so in this case we simply put $\left.\mathcal{B}\right|_{W \Gamma(u, v)}:=\left.\mathcal{B}\right|_{b W \Gamma(u, v)}$. For $(u, v)=\left(v_{\text {init }}, v_{\text {fin }}\right)$, let $\left.\mathcal{B}\right|_{W \Gamma\left(v_{\text {init }}, v_{\text {fin }}\right)}$ be an arbitrary extension of $\left.C \mathcal{A}\right|_{b W \Gamma\left(v_{\text {init }}, v_{\text {fin }}\right)}$. Such an extension exists, because $W \Gamma\left(v_{\text {init }}, v_{\text {fin }}\right)$ is, by Proposition 2.15, a cone over $b W \Gamma\left(v_{\text {init }}, v_{\text {fin }}\right)$ and $\left.C \mathcal{A}\right|_{b W \Gamma\left(v_{\text {init }}, v_{\text {fin }}\right)}$ is, by assumption, homotopic to a constant map. It is obvious that $\mathcal{B}$ defined in this way is a functor - 
since all decomposable morphisms of $W \Gamma$ belong to $b W \Gamma$, there are no categorial constraints on the extension $\mathcal{B}$. Equation (3.7) is clearly also satisfied.

The opposite implication - that the existence of a functor $\mathcal{B}: W \Gamma \rightarrow \mathcal{T}_{*}$ satisfying (3.7) implies the vanishing of $\langle\langle\mathcal{A}\rangle\rangle-$ also follows directly from Proposition 2.15.

It is easy to see that (3.7) is equivalent to

$$
\pi \circ \mathcal{B}(\sigma(f))=\mathcal{A}(f)
$$

for each $f \in \Gamma$. We can now formulate and prove the main result of this section:

3.8. Theorem. The homotopy operation $\langle\langle\mathcal{A}\rangle\rangle$ vanishes (and in particular, is defined) if and only if $\mathcal{A}$ has a rectification, so it is precisely the last obstruction to rectifying $\mathcal{A}$.

Proof. The proof is an immediate consequence of Theorem 2.18. Let $\mathbf{X}$ denote, throughout this proof, the $V$-graded space defined by $X(v):=\mathcal{A}(v)$, for $v \in V$.

Let us prove first that the vanishing of $\langle\langle\mathcal{A}\rangle\rangle$ implies the existence of a rectification. By Proposition 3.6, vanishing of $\langle\langle\mathcal{A}\rangle\rangle$ is equivalent to the existence of a functor $\mathcal{B}$ : $W \Gamma \rightarrow \mathcal{T}_{*}$ such that $[\mathcal{B}(\sigma(f))]=\mathcal{A}(f)$ for each arrow $f: u \rightarrow v \in \Gamma$. The functor $\mathcal{B}$ is a $W \Gamma$-structure on the $V$-graded space $\mathbf{X}$ and Theorem 2.18(a) provides a $\Gamma$-space $\mathbf{Y}$ defined by a functor $F: \Gamma \rightarrow \mathcal{T}_{*}$, together with a homotopy equivalence $\mathbf{h}=\left\{h_{u}: \mathcal{B}(u) \rightarrow F(u) \mid u \in V\right\}$, for which diagram (2.19) commutes up to homotopy, that is

$$
[F(f)] \circ\left[h_{u}\right]=\left[h_{v}\right] \circ[\mathcal{B}(\sigma(f))]
$$

in ho $\mathcal{T}_{*}$. Since $[\mathcal{B}(\sigma(f))]=\mathcal{A}(f)$, this means that $F: \Gamma \rightarrow \mathcal{T}_{*}$ is a rectification of $\mathcal{A}$.

To prove that the existence of a rectification implies the vanishing of $\langle\langle\mathcal{A}\rangle\rangle$, let $F: \Gamma \rightarrow \mathcal{T}_{*}$ be such a rectification - that is, a $V$-graded $\Gamma$-space $\mathbf{Y}=\{F(v) \mid v \in V\}$, together with a system $\left\{h_{u}: \mathcal{A}(u) \rightarrow F(u) \mid u \in V\right\}$ such that

$$
[F(f)] \circ\left[h_{u}\right]=\left[h_{v}\right] \circ \mathcal{A}(f)
$$

for each $f: u \rightarrow v \in \Gamma$. By Theorem 2.18(b), there exists a $W \Gamma$-structure $\mathcal{B}$ on $\mathbf{X}$ such that $[F(f)] \circ\left[h_{u}\right]=\left[h_{v}\right] \circ[\mathcal{B}(\sigma(f))]$. Combining this with $(3.9)$ and using the invertibility of $\left[h_{v}\right]$ in ho $\mathcal{T}_{*}$ we see that $[\mathcal{B}(\sigma(f))]=\mathcal{A}(f)$ for each $f$, therefore $\langle\langle\mathcal{A}\rangle\rangle=0$, by Proposition 3.6. 
3.10. A pointed version. Since the category $\mathcal{T}_{*}$ is pointed, the null map $*$ has a special status. We may therefore require that whenever $\mathcal{A}(f)=*$ (the null class), we take $B \mathcal{A}(f)=*$ to be the null map itself (rather than just nullhomotopic), and more generally that for any cubical face $C[\Psi]$ of $W \Gamma$, if the corresponding map on the boundary $\partial C[\Psi] \times X \rightarrow Y$ is the null map, then the extension to $C[\Psi] \times X \rightarrow Y$ also be the null map. In this case we say that the corresponding complete data are pointed.

This is in fact the most common sense in which the term "secondary (or higher order) homotopy operation" is used. As we shall see in $\$ 3.19$ below, there are practical reasons why this is the prefered version.

Note that in this case one can actually replace the basis $\mathcal{P}=b W \Gamma$ by a simplified basis $\hat{\mathcal{P}}=\widehat{b W \Gamma}$, in which we collapse all faces indexed by at least one zero map to a single point. Of course, the resulting cell complex may no longer be a polyhedron; see Figure 3.13 in Example 3.20 below.

3.11. Relative operations. More generally, we may choose to specify a partial rectification of the diagram $\mathcal{A}: \Gamma \rightarrow$ ho $\mathcal{T}_{*}$, in the sense that there is a sub-diagram $\Gamma^{\prime} \subset \Gamma$ equipped with a specific lift of $\left.\mathcal{A}\right|_{\Gamma^{\prime}}$ to a functor $F^{\prime}: \Gamma^{\prime} \rightarrow \mathcal{T}_{*}$. In this case again we assume that the extensions to the appropriate faces of $b \mathcal{P}_{\Gamma}$ are constant, and call the corresponding subset of $\left[\mathcal{A}\left(v_{\text {init }}\right) \rtimes b \mathcal{P}, \mathcal{A}\left(v_{\text {fin }}\right)\right]\left(\right.$ rel $\left.\mathcal{A}\left(v_{\text {init }}\right) \rtimes b \mathcal{P}_{\Gamma^{\prime}}\right)$ a relative higher homotopy operation.

3.12. Example. The classical example of the Toda bracket fits into the variant of $\S 3.10$, since in the usual description we require certain of the maps constituting the full data for this operation to be specifically the null map (rather than allowing any null-homotopic map, as one would in a fully homotopy-invariant description):

Recall that the usual Toda bracket is defined whenever one has three composable maps $X \stackrel{\gamma}{\rightarrow} Y \stackrel{\beta}{\rightarrow} Z \stackrel{\alpha}{\rightarrow} W$, and each of the compositions $\alpha \circ \beta$ and $\beta \circ \gamma$ is nullhomotopic. We thus have a lattice $\Gamma$ as in Figure 3.13, with four commuting triangles marked $F$, $\mathrm{G}, \mathrm{H}$ and $\mathrm{K}$. Note that this is not a planar graph: the two outer (angled) edges, both marked $f_{*}$, should be identified.

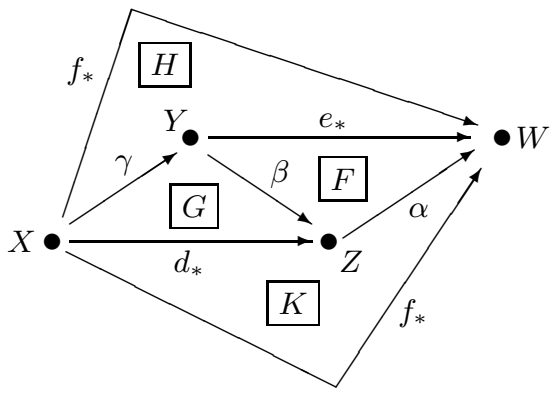

Figure 3.13. Lattice for Toda bracket 
The homotopy classes of $\alpha, \beta$ and $\gamma$ as above define initial data $\mathcal{A}$ as indicated by the diagram, with $f_{*}, e_{*}$ and $d_{*}$ being null maps. The associated polyhedron $\mathcal{P}$ is a square, as in Figure 3.14, where $b W \Gamma(X, W)$ consists of the upper and right edges of the square.

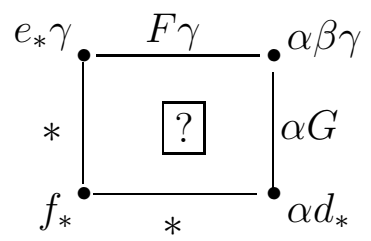

FiguRE 3.14. Square for Toda bracket

Since $b W \Gamma(X, Z)$ is the interval with endpoints $\beta \circ \gamma$ and $d_{*}$, and $b W \Gamma(Y, W)$ is the interval with endpoints $\alpha \circ \beta$ and $e_{*}$, complete data are given by a choice of homotopies $F$ and $G$ corresponding to commutative triangles $G$ and $F$; the homotopies corresponding to triangles $\mathrm{H}$ and $\mathrm{K}$ are assumed to be trivial. The fill-in marked ? exists (with trivial homotopies along the edges marked "*") if and only if the corresponding Toda bracket $\langle\langle\mathcal{A}\rangle\rangle \subset\left[X \rtimes S^{1}, W\right]$ vanishes.

3.15. Conditions for existence. Given initial data $\mathcal{A}: \Gamma \rightarrow$ ho $\mathcal{T}_{*}$, it is natural to try to construct complete data $C \mathcal{A}: b W \Gamma \rightarrow \mathcal{T}_{*}$ and a functor $\mathcal{B}: W \Gamma \rightarrow \mathcal{T}_{*}$ inductively, using the skeletal filtration of $b W \Gamma$.

3.16. Definition. For $n \geq 0$, an $n$-realization of the initial data $\mathcal{A}$ is a functor $\overline{\mathcal{A}}_{n}: \operatorname{sk}_{n} b W \Gamma \rightarrow \mathcal{T}_{*}$ such that $\left[\overline{\mathcal{A}}_{n}(f)\right]=\mathcal{A}(f)$ in ho $\mathcal{T}_{*}$ for any arrow $f$ in $\Gamma \backslash\left\{\phi_{\max }\right\} \subset$ $\mathrm{sk}_{0} b W \Gamma$.

It follows from the freeness of the category $\mathrm{sk}_{0} W \Gamma(\$ 2.14)$ that there always exists a 0 -realization $\overline{\mathcal{A}}_{0}$. It sends any partially parenthesized sequence representing a vertex of $\operatorname{sk}_{0} b W \Gamma-$ such as $f g(h k \ell) m$ - to the corresponding chain in $\mathcal{T}_{*}$ - e.g., $\overline{\mathcal{A}}_{0} f \overline{\mathcal{A}}_{0} g \overline{\mathcal{A}}_{0}(h \circ$ $k \circ \ell) \overline{\mathcal{A}}_{0} m$. Observe also that the initial data $\mathcal{A}$ are uniquely determined by any of 0 -realizations of $\mathcal{A}$.

Using the above terminology, we can also say that complete data are given by a functor $C \mathcal{A}: b W \Gamma \rightarrow \mathcal{T}_{*}$ extending some 0-realization of $\mathcal{A}$. In the same vein, the vanishing of $\langle\langle\mathcal{A}\rangle\rangle$ is equivalent to the existence of a functor $\mathcal{B}: W \Gamma \rightarrow \mathcal{T}_{*}$ that extends some 0realization $\overline{\mathcal{A}}_{0}$. Indeed, for such a functor we have $[\mathcal{B}(f)]=\left[\overline{\mathcal{A}}_{0}(f)\right]=\mathcal{A}(f)$ whenever $f \in \Gamma \backslash\left\{\phi_{\max }\right\}$, while $\left[\mathcal{B}\left(\phi_{\max }\right)\right]=\mathcal{A}\left(\phi_{\max }\right)$ follows from the fact that $W \Gamma\left(v_{\text {init }}, v_{\text {fin }}\right)$ is the cone over $b W \Gamma\left(v_{\text {init }}, v_{\text {fin }}\right)$ with vertex $\left\langle\phi_{\max }\right\rangle$. Compare also Proposition 3.6 and its proof.

Let us show that the success or failure of the induction does not depend on the first step of the construction: 
3.17. Proposition. Suppose that there exists a 0 -realization $\overline{\mathcal{A}}_{0}: \mathrm{sk}_{0} b W \Gamma \rightarrow \mathcal{T}_{*}$ which can be extended to a functor $\mathcal{B}: W \Gamma \rightarrow \mathcal{T}_{*}$. Then an arbitrary 0 -realization $\overline{\mathcal{A}}_{0}^{\prime}$ can be extended to some functor $\mathcal{B}^{\prime}: W \Gamma \rightarrow \mathcal{T}_{*}$.

Proof. Let $\overline{\mathcal{A}}_{0}: \mathrm{sk}_{0} b W \Gamma \rightarrow \mathcal{T}_{*}$ be a 0-realization of $\mathcal{A}, \mathcal{B}: W \Gamma \rightarrow \mathcal{T}_{*}$ its functorial extension and $\overline{\mathcal{A}}_{0}^{\prime}: \mathrm{sk}_{0} b W \Gamma \rightarrow \mathcal{T}_{*}$ another 0 -realization of $\mathcal{A}$. Since, by definition,

$$
\left[\overline{\mathcal{A}}_{0}(f)\right]=\left[\overline{\mathcal{A}}_{0}^{\prime}(f)\right]=\mathcal{A}(f)
$$

in ho $\mathcal{T}_{*}$, for each $f \in \Gamma \backslash\left\{\phi_{\max }\right\} \subset \mathrm{sk}_{0} b W \Gamma$, there exists a homotopy through functors $H_{t}: \operatorname{sk}_{0} b W \Gamma \rightarrow \mathcal{T}_{*}$ from $\overline{\mathcal{A}}_{0}$ to $\overline{\mathcal{A}}_{0}^{\prime}$. Indeed, choose $H_{t}$ arbitrarily on generators $f \in \Gamma \backslash\left\{\phi_{\max }\right\}$ - this is possible by (3.18) - and then extend $H_{t}$ functorially using the freeness of $\mathrm{sk}_{0} b W \Gamma$ (Remark 2.14).

Because $\mathrm{sk}_{0} b W \Gamma$ is an admissible subcategory of $W \Gamma$, we may apply Proposition 2.20 to obtain an extension $F_{t}: W \Gamma \rightarrow \mathcal{T}_{*}$ of $\mathcal{B}$ and $H_{t}$. Then $\mathcal{B}^{\prime}:=F_{1}: W \Gamma \rightarrow \mathcal{T}_{*}$ is an extension of $\overline{\mathcal{A}}^{\prime}$.

3.19. Remark. In the case of a pointed higher homotopy operation, in the sense of $\$ 3.10$, we are often in the situation of Example 3.12, in that one (or more) of the vertices of $b \mathcal{P}$ is taken by $\mathcal{A}$ to the null map. This implies that we may in fact think of the higher homotopy operation $\langle\langle\mathcal{A}\rangle\rangle$ as comprising a subset of $\left[\mathcal{A} v_{\text {init }} \wedge b \mathcal{P}, \mathcal{A} v_{\text {fin }}\right]_{\text {ho } \mathcal{T}_{*}}$ - where we choose the above vertex of $b \mathcal{P}$ as the base point. This is useful when $b \mathcal{P}$ is a sphere, up to homotopy, since in that case the higher operations take value in an (abelian) group.

3.20. Example. Longer sequences of composable maps yield higher Toda brackets, as in Wa, Mo. For instance, given the lattice $L_{4}=\left\langle v_{\text {init }} \stackrel{k}{\rightarrow} v_{4} \stackrel{h}{\rightarrow} v_{3} \stackrel{g}{\rightarrow} v_{2} \stackrel{f}{\rightarrow} v_{\text {fin }}\right\rangle$ of Example 2.11, we obtain a three-dimensional cube as in Figure 2.12 for $W L_{4}$, with basis as in Figure 2.17. However, if we assume that $f \circ g, g \circ h$, and $h \circ k$ are all null, and we are interested in the pointed third-order operation $(\$ 3.10)$, we may replace $\mathcal{P}=b W L_{4}$ by the simplified basis $\widehat{\mathcal{P}}=\widehat{b W \Gamma}$ of Figure 3.21.

If we carry out the indicated identifications (i.e., collapse the boundary of the outer triangle to a point), we obtain a 2-sphere, so we are indeed in the situation describe in Remark 3.19.

3.22. Extending $k$-realizations. Assume given initial data $\mathcal{A}: \Gamma \rightarrow$ ho $\mathcal{T}_{*}$; then we always have some extension of any 0-realization $\overline{\mathcal{A}}_{0}: \mathrm{sk}_{0} b W \Gamma \rightarrow \mathcal{T}_{*}$ to $\mathrm{sk}_{1} b W \Gamma$, since this merely involves choosing homotopies between maps which are homotopic by definition. However, this is not true of higher skeleta: a given $(k-1)$-realization may not extend to a $k$-realization, and it may extend in more than one way; as expected, we have an obstruction theory. Rather than describing it in detail, we shall sketch only the part that is relevant to higher homotopy operations; in particular, we disregard the obstructions for distinguishing between different extensions. 


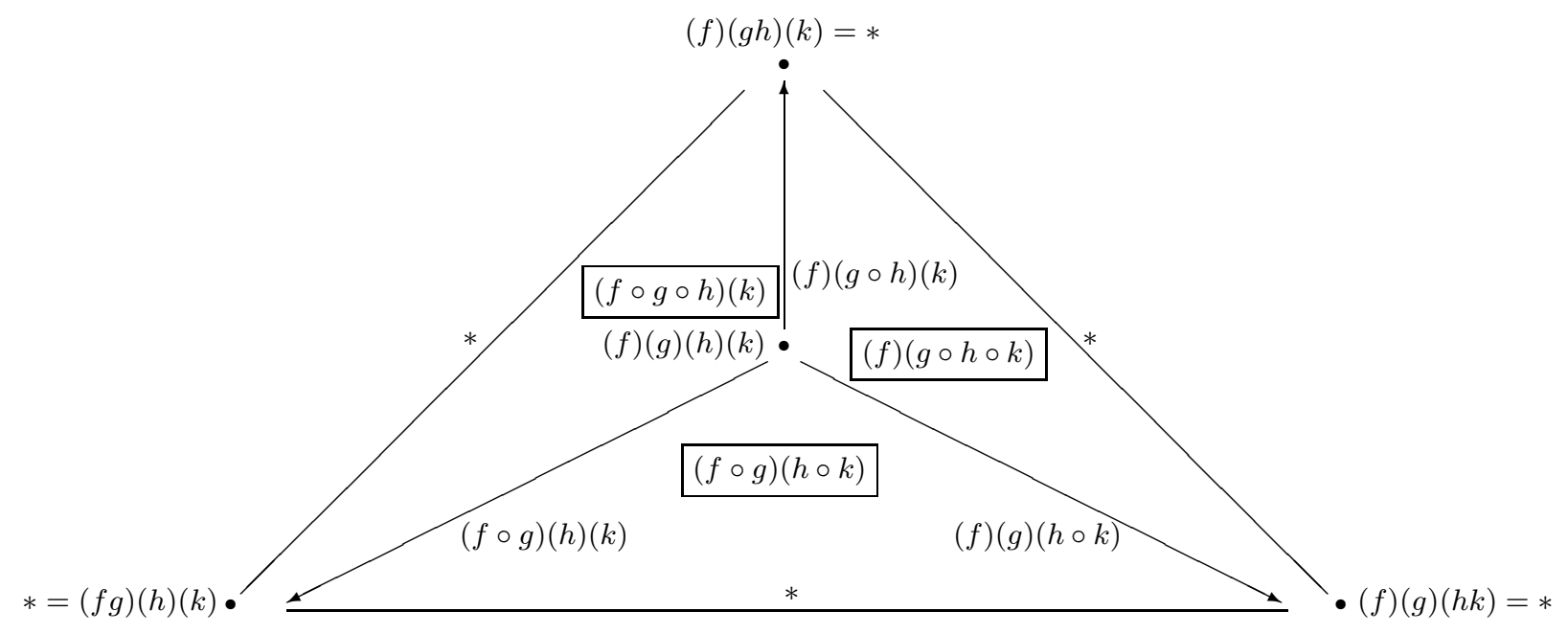

Figure 3.21. Simplified basis for $\widehat{b W L}_{4}$

Note that, given what we know about $\overline{\mathcal{A}}_{0}$, it is natural to make two requirements for our induction:

3.23. Assumption. At the $k$-th stage of the induction $(k \geq 1)$, we assume that:

(a) we have fixed a $(k-1)$-realization $\overline{\mathcal{A}}_{k-1}: \operatorname{sk}_{k-1} b W \Gamma \rightarrow \mathcal{T}_{*}$;

(b) $\overline{\mathcal{A}}_{k-1}$ can be extended over $\mathrm{sk}_{k} b W \Gamma$ - although we do not choose a particular $k$-realization $\overline{\mathcal{A}}_{k}$.

Note also that if $n=\operatorname{dim} b W \Gamma$, the $n$-th induction assumption is precisely what we want in order for $\langle\langle\mathcal{A}\rangle\rangle$ to be defined; the various choices of $\overline{\mathcal{A}}_{n}$ (for all possible choices of $\overline{\mathcal{A}}_{n-1}$ ) then define its members.

3.24. Definition. Given a maximal chain

$$
\Phi=\left\langle v_{\text {init }}=v_{n} \stackrel{f_{n}}{\longrightarrow} v_{n-1} \stackrel{f_{n-1}}{\longrightarrow} \cdots \stackrel{f_{2}}{\longrightarrow} v_{1} \stackrel{f_{1}}{\longrightarrow} v_{0}=v_{\text {fin }}\right\rangle
$$

of $\Gamma$, every basis point $\left\langle\begin{array}{llllllll}f_{1} & \circ_{t_{1}} & f_{2} & \circ_{t_{2}} & \cdots & f_{n-1} & \circ_{t_{n-1}} & f_{n}\end{array}\right\rangle$ in the corresponding subcomplex $C[\Phi] \cap b W \Gamma$ is in $\operatorname{sk}_{k} b W \Gamma$ if and only if it has at least $(n-k-1)$ coordinates equal to 1 or 0 . Each choice of a subset $M \subset\{1,2, \ldots, n-1\}$ (those of the coordinates which we wish to set equal to 1 ) yields a partition of $\Phi$ into $r$ disjoint subchains: $\Phi=\Psi^{1} \Psi^{2} \cdots \Psi^{r}$.

To each proper subchain $\Psi^{i}=\left\langle v_{\text {init }}^{i} \stackrel{f_{n_{i}+1}^{i}}{\longrightarrow} v_{n_{i}}^{i} \stackrel{f_{n_{i}}^{i}}{\longrightarrow} \cdots \stackrel{f_{2}^{i}}{\longrightarrow} v_{1}^{i} \stackrel{f_{1}^{i}}{\longrightarrow} v_{\text {fin }}^{i}\right\rangle$ of length $n_{i}+1$, we associate the $n_{i}$-dimensional cube $C\left[\Psi^{i}\right]$ - necessarily in $b W \Gamma$, because of the properness - and let $C^{M}[\Phi]$ denote the subcomplex $C\left[\Psi^{1}\right] \times C\left[\Psi^{2}\right] \times \cdots \times C\left[\Psi^{r}\right]$ of $b W \Gamma-$ itself a cube.

If all but one of $\Psi^{1}, \Psi^{2}, \ldots, \Psi^{r}$ is of length 1 - so that the corresponding factors $C\left[\Psi^{i}\right]$ are zero-dimensional - we say that $C^{M}[\Phi]$ is indecomposable. Denote by $\Im^{k}$ the collection of all indecomposable $k$-cubes of $b W \Gamma$ (for all maximal chains $\Phi$ ). 
Let $\mathcal{I}_{k}$ denote the collection of all unordered pairs $\left\{C_{i}, C_{j}\right\}$ of indecomposable $(k+1)$-cubes of $b W \Gamma$ such that $C_{i, j}:=C_{i} \cap C_{j}$ is $k$-dimensional.

3.25. Example. For $\Gamma=L_{4}$ as in Example 2.11 above, with $b W L_{4}$ as in Figure 2.17, we have three 2-dimensional cubes, of which the first two are indecomposable:

(a) The left quadrilateral is $C_{1}:=C\left[\Psi^{1}\right] \times C\left[\Psi^{2}\right]$, where $\Psi^{1}:=\left\langle v_{\text {init }} \stackrel{k}{\rightarrow} v_{4}\right\rangle$ (so $C\left[\Psi^{1}\right]$ is a point) and $\Psi^{2}=\left\langle v_{4} \stackrel{h}{\rightarrow} v_{3} \stackrel{g}{\rightarrow} v_{2} \stackrel{f}{\rightarrow} v_{\text {fin }}\right\rangle$.

(b) The upper right square is $C_{2}:=C\left[\Psi^{3}\right] \times C\left[\Psi^{4}\right]$, for $\Psi^{3}=\left\langle v_{\text {init }} \stackrel{k}{\rightarrow} v_{4} \stackrel{h}{\rightarrow} v_{3} \stackrel{g}{\rightarrow} v_{2}\right\rangle$ and $\Psi^{4}=\left\langle v_{2} \stackrel{f}{\rightarrow} v_{\text {fin }}\right\rangle$.

(c) The lower right quadrilateral decomposes as the product of two 1-dimensional subcubes, corresponding to $v_{\text {init }} \stackrel{k}{\rightarrow} v_{4} \stackrel{h}{\rightarrow} v_{3}$ and $v_{3} \stackrel{g}{\rightarrow} v_{2} \stackrel{f}{\rightarrow} v_{\text {fin }}$, respectively.

$\mathcal{I}_{2}$ consists of the single pair $\left\{C_{1}, C_{2}\right\}$, so $C_{1,2}=C_{1} \cap C_{2}$ is the vertical 1-cube denoted by $(f)(g \circ h)(k)$ in Figure 2.17.

3.26. Conditions for the induction step. Let us assume that 3.23 holds for $k$, so $\overline{\mathcal{A}}_{k-1}$ has been chosen. Note that because $\overline{\mathcal{A}}_{k-1}: \mathrm{sk}_{k-1} b W \Gamma \rightarrow \mathcal{T}_{*}$ is a functor, it extends uniquely to all decomposable cubes $C^{M}[\Phi]=C\left[\Psi^{1}\right] \times C\left[\Psi^{2}\right] \times \cdots \times C\left[\Psi^{r}\right]$ as above, as long as each $C\left[\Psi^{i}\right]$ has dimension $\leq k-1$ (and it can be extended if $\operatorname{dim}\left(C\left[\Psi^{i}\right]\right) \leq k$ for all $\left.i\right)$

We want to choose a $k$-realization $\overline{\mathcal{A}}_{k}$ in such a way that it has some extension to the $(k+1)$-skeleton of $b W \Gamma$. By the above, it is enough to extend it to the set $\Im^{k+1}=\left\{C_{1}, \ldots, C_{\ell}\right\}$ of all indecomposable $(k+1)$-cubes for $\Gamma$, where $C_{i}=W \Gamma_{i}$ for some sublattice $\Gamma_{i}$ of $\Gamma$ (isomorphic to $L_{k+2}$ of $\S 2.2$ ), so that $b W \Gamma_{i}$ is $k$-dimensional.

Consider the initial data $\mathcal{A}_{i}:=\left.\mathcal{A}\right|_{\Gamma_{i}}$ for each $1 \leq i \leq \ell$. Clearly, Assumption 3.23(b) (for $k$ ) is needed in order for each of the higher homotopy operations $\left\langle\left\langle\mathcal{A}_{1}\right\rangle\right\rangle, \ldots,\left\langle\left\langle\mathcal{A}_{\ell}\right\rangle\right\rangle$ to even be defined, (since we need an extension to $b W \Gamma_{i}$ for each $i$ ); their vanishing is necessary in order for 3.23(a) to hold for $(k+1)$, since it must be possible to extend the new $\overline{\mathcal{A}}_{k}$ over the $(k+1)$-sekeleton.

However, a sufficient condition for 3.23 to hold for $(k+1)$ is that the operations $\left\langle\left\langle\mathcal{A}_{i}\right\rangle\right\rangle$ vanish coherently - which means, essentially, that they can be made to vanish by choices of realizations which agree on the common $k$-dimensional faces of the different cubes $C_{i}$ (and which equal the given $\overline{\mathcal{A}}_{k-1}$ on the $(k-1)$-skeleta of these faces). In order to formulate this condition precisely - without specifying a global $\overline{\mathcal{A}}_{k}$ for all of $b W \Gamma$ - we need the following

3.27. Definition. For $k \geq 1$, let $C$ be a $k$-cube (we have in mind any of the common facets of two indecomposable $(k+1)$-cubes), with boundary $\partial C=\operatorname{sk}_{k-1} C$, and let $f: X \rtimes \partial C \rightarrow Y$ be a map. Note that the space obtained by identifying the boundaries $\partial C$ in two different copies of $C$ is canonically homeomorphic to the unreduced suspension 
$\Sigma \partial C$, so each pair of extensions $F_{1}, F_{2}: X \rtimes C \rightarrow Y$ of $f$ defines a map $D_{F_{1}, F_{2}}$ : $X \rtimes \Sigma \partial C \rightarrow Y$, whose homotopy class (relative to $X \rtimes \partial C$ ) depends only on the homotopy classes of $F_{1}$ and $F_{2}$ relative to $X \rtimes \partial C$. This class is called the difference obstruction for $F_{1}$ and $F_{2}$ relative to $f$, and is denoted by

$$
\delta_{f}\left(F_{1}, F_{2}\right) \in[X \rtimes \Sigma \partial C, Y(\operatorname{rel} X \rtimes \partial C)] .
$$

3.28. Definition. Assume that for each $C_{i}=W \Gamma_{i}(i=1, \ldots, \ell)$ in $\Im^{k+1}$ we have some extension $F_{i}: W \Gamma_{i} \rightarrow \mathcal{T}_{*}$ of $\left.\overline{\mathcal{A}}_{k-1}\right|_{\mathrm{sk}_{k-1} C_{i}}$ (for a $(k-1)$-realization $\overline{\mathcal{A}}_{k-1}$ as in \$3.23). Note that such an $F_{i}$ exists if and only if the corresponding higher operation $\left\langle\left\langle\mathcal{A}_{i}\right\rangle\right\rangle$ of $\$ 3.26$ vanishes.

Then the obstruction sequence determined by $\left(F_{i}\right)_{i=1}^{\ell}$ is the finite sequence

$$
\Delta\left(F_{i}\right)_{i=1}^{\ell}:=\prod_{\left\{C_{i}, C_{j}\right\} \in \mathcal{I}_{k}} \delta_{\overline{\mathcal{A}}_{k-1}}\left(\left.F_{i}\right|_{C_{i, j}},\left.F_{j}\right|_{C_{i, j}}\right) .
$$

The obstruction sequence $\Delta\left(F_{i}\right)_{i=1}^{\ell}$ is said to vanish if each of its components $\delta_{\overline{\mathcal{A}}_{k-1}}\left(\left.F_{i}\right|_{C_{i, j}},\left.F_{j}\right|_{C_{i, j}}\right)$ is null.

Note that these classes depend only on the homotopy classes of $\left.F_{i}\right|_{C_{i, j}}$ and $\left.F_{j}\right|_{C_{i, j}}$ relative to $\mathrm{sk}_{k-1} C_{i, j}$. Evidently:

3.29. Theorem. Assume given initial data $\mathcal{A}$; if $\overline{\mathcal{A}}_{k-1}: \mathrm{sk}_{k-1} b W \Gamma \rightarrow \mathcal{T}_{*}$ is a $(k-1)$ realization which can be extended to a k-realization, then $\overline{\mathcal{A}}_{k-1}$ extends further to a $(k+1)$-realization if and only if it has some vanishing obstruction sequence.

3.30. Remark. This theorem is of little use, if viewed as an obstruction theory for successively constructing $k$-realizations of $\mathcal{A}$. It should be thought of rather as an attempt to make some sense of the claim that "an $n$-th order homotopy operation is defined if and only if all lower order operations vanish coherently." The classes in $\delta_{\overline{\mathcal{A}}_{k-1}}$ should be thought of as the obstructions to coherence; the vanishing of the individual operations is implicit in Definition 3.28.

3.31. Example. In order for the "long Toda bracket" $\langle\langle\mathcal{A}\rangle\rangle=\langle f, g, h, k\rangle$ of Example 3.20 to be defined, the two (ordinary) Toda brackets $\langle f, g, h\rangle$ and $\langle g, h, k\rangle-$ corresponding to $C_{1}$ and $C_{2}$ of Example 3.25, respectively - must vanish. (We may disregard the lower quadrilateral (or triangle), which is decomposable, and thus represents a "product" of ordinary nullhomotopies $f \circ g \sim *$ and $h \circ k \sim *$.)

By Theorem 3.29, the only difference obstruction corresponds to $C_{1,2}$ of Example 3.25 - the edge $(f)(g \circ h)(k)$ in Figure 3.13. The vanishing of $\delta_{\overline{\mathcal{A}}_{1}}$ is clearly equivalent to the choosing of homotopic nullhomotopies for $g \circ h \sim *$ in defining the two vanishing elements in $\langle f, g, h\rangle$ and $\langle g, h, k\rangle$, respectively (relative to fixed choices of $g$ and $h$ ). 


\section{Families of polytopes}

We have defined higher homotopy operations for any lattice $\Gamma$, as the (final) obstruction to rectifying diagrams $\mathcal{A}: \Gamma \rightarrow$ ho $\mathcal{T}_{*}$. In this generality there is very little structure to them. However, in most cases of interest $\Gamma$ will have certain "symmetries" which will simplify the description of the faces of $W \Gamma\left(v_{\text {init }}, v_{\text {fin }}\right)$ and ensure that $\mathcal{P}=W \Gamma\left(v_{\text {init }}, v_{\text {fin }}\right)$ will be combinatorially equivalent to an $n$-dimensional polytope (i.e., convex polyhedron in $\mathbb{R}^{n}$ ), with basis $b W \Gamma$ equivalent to its boundary $\partial \mathcal{P}$, which is thus homeomorphic to a sphere.

This is important because of Remark 3.19 above, since in that situation the corresponding higher operations actually take value in appropriate track groups (which are abelian, for $n \geq 3$ ), and the indeterminacy may often be described in terms of appropriate cosets, as for the classical Toda bracket (cf. [T2, Ch. I]).

4.1. Definition. A family of polytopes is a sequence $\mathcal{F}=\left(P_{n}\right)_{n=0}^{\infty}$ of polytopes, starting with $P_{0}=\{\mathrm{pt}\}$, such that $\operatorname{dim}\left(P_{n}\right)=n$, and each facet of $P_{n}$ is isomorphic to some product of lower dimensional polytopes from $\mathcal{F}$.

4.2. Examples. Many familiar examples of polytopes fit into such families:

1. The family $\Delta$ of standard $n$-simplices $\{\Delta[n]\}_{n=0}^{\infty}$.

2. The family $\mathcal{C} u$ of $n$-cubes $\left\{[0,1]^{n}\right\}_{n=0}^{\infty}$.

3. The family $\mathcal{A} s s=\left(K_{n}\right)_{n=0}^{\infty}$ of associahedra, due to Stasheff (cf. [St] $)$.

4. The family $\mathcal{P e r m}=\left(P e_{n}\right)_{n=0}^{\infty}$ of permutohedra, apparently due to Schoute at the beginning of the twentieth century (cf. $[\mathbf{S c}]$ ), where $P e_{n}=P e_{n}\left(a_{0}, \ldots, a_{n}\right)$ is defined to be the convex hull of the $(n+1)$ ! points $\left(\sigma\left(a_{0}\right), \sigma\left(a_{1}\right), \ldots, \sigma\left(a_{n}\right)\right) \in \mathbb{R}^{n+1}$, indexed by permutations $\sigma \in \Sigma_{n+1}$, where $a_{0}, \ldots, a_{n}$ are any distinct real numbers (though we usually take $\left(a_{0}, \ldots, a_{n}\right)=(0,1,2, \ldots, n)$ ).

Note that in all of these examples we can find a more economical description for $\mathcal{P}$ than the cubical or simplicial ones described above for general $\Gamma$ - for example, instead of triangulating the 2-dimensional associahedron using ten 2-simplices, or five 2-cubes, we can think of it as just a single pentagon. Such "minimal models" are clearly useful, but there appears to be no canonical way to obtain them, in general.

4.3. Permutohedra. The family of permutohedra $\operatorname{Perm}=\left(P e_{n}\right)_{n=0}^{\infty}$, which is in some sense the universal family of polytopes, is associated to the category $\mathbb{N}_{+}$of \$1.1. The family Perm has appeared in a number of homotopy theoretic contexts - in the work of Milgram [Mi], §4], Stasheff [St3, §11], Baues [Ba, III, (4.5)], and others.

If we let $\Gamma$ denote the full subcategory of $\mathbb{N}_{+}^{o p}$ with objects $\{[-\mathbf{1}],[\mathbf{0}], \ldots,[\boldsymbol{n}]\}$, then we have maps $d_{i}^{k}:[\boldsymbol{k}] \rightarrow[\boldsymbol{k}-\mathbf{1}]$ for $0 \leq i \leq k \leq n$, with

$$
d_{i}^{k-1} d_{j}^{k}=d_{j-1}^{k-1} d_{i}^{k} \text { for all } 0 \leq i<j \leq k \leq n
$$


(where $d_{0}^{0}:=\varepsilon$ is the augmentation).

The $n$-dimensional permutohedron $P e_{n}$ is then a minimal model for $\mathcal{P}$, and the simplicial complex $\operatorname{Map}([\boldsymbol{n}],[-\mathbf{1}])$ which triangulates $W \Gamma$ (see $\S 2.21$ ) is the first barycentric subdivision of the obvious triangulation of $P e_{n}$. The isomorphism may be described explicitly as follows:

Let $\Phi^{\mathrm{Id}}$ denote the (maximal) $(n+1)$-chain of $\Gamma$, obtained by writing the unique map $\varphi: v_{\text {init }}=[\boldsymbol{n}] \rightarrow[-\mathbf{1}]=v_{\text {fin }}$ of $\Gamma$ in standard form as $d_{0} d_{1} d_{2} \cdots d_{n}$. To the vertex $v_{\sigma}$ $\left(\sigma \in \Sigma_{n+1}\right)$ of $P e_{n}$ we then associate the vertex indexed by the $(n+1)$-chain $\Phi^{\sigma}$ of $\Gamma$ obtained from $\Phi^{\mathrm{Id}}$ by carrying out the permutation $\sigma$ on the maps $\left(d_{0}, d_{1}, d_{2}, \ldots, d_{n}\right)$ and applying (4.4) for each adjacent transposition.

There is an edge $e$ in $P e_{n}$ connecting $v_{\sigma}$ with $v_{\tau}$ whenever $\tau$ is obtained from $\sigma$ by an adjacent transposition $(i, i+1)$; the barycenter of $e$ is indexed by the $n$-chain obtained from $\Phi^{\sigma}$ (or $\Phi^{\tau}$ ) by composing the two maps at the $(i+1)$-st node.

4.5. Remark. More geometrically, we can think of $\Gamma$ as the lattice the $k$-simplices $\Delta[k]$ $(0 \leq k \leq n)$, with morphisms consisting of all possible inclusions of faces.

Alternatively, consider the lattice $\Gamma^{\prime}$ of all subsets of $[\boldsymbol{n}]=\{0,1,2, \ldots, n\}$, with initial object $\emptyset$ and terminal object $[\boldsymbol{n}]$; which is equivalent, of course, to the lattice of all faces of the simplicial complex $\Delta[n]$ (with morphisms again the inclusions). Viewed in this way, $P e_{n}$ will be called the face-polyhedron of $\Delta[n]$. Many other interesting families of polyhedra can be constructed similarly.

The lattice $\Gamma$ defined above (as well as its opposite $\Gamma^{o p}$ ) is obtained from $\Gamma^{\prime}$ by successively identifying all subsets of $[\boldsymbol{n}]$ of cardinality $k$ to a single node $[\boldsymbol{k}]$, without changing the morphisms. More precisely, if $k<\ell, B$ is some subset of $[\boldsymbol{n}]$ of cardinality $\ell$, and $A_{1}, \ldots, A_{r}$ are the $k$-subsets of $B$, then $\operatorname{Hom}_{\Gamma}([\boldsymbol{k}],[\boldsymbol{l}]):=\bigcup_{i=1}^{r} \operatorname{Hom}_{\Gamma^{\prime}}\left(A_{i}, B\right)$.

As a result, the corresponding cubical categories $W \Gamma^{\prime}$ and $W \Gamma$ have homeomorphic mapping spaces - and in particular, the same total mapping space $\mathcal{P}=\mathcal{P}_{\Gamma^{\prime}} \cong \mathcal{P}_{\Gamma}$. However, for our purposes we do need to consider the (distinct) category structures on $W \Gamma^{\prime}$ and $W \Gamma$.

Of course, this "condensed" construction works only because all $k$-dimensional faces of the simplex are 'the same,' (i.e., the group of automorphisms act transitively on the set of $k$-dimensional faces, for all $k$ ). For a general polyhedron, we expect different faces to correspond to different nodes in the lattice of faces $\Gamma$. However, if we are only interested in a minimal model for $W \Gamma$, as in the case of the permutohedron, the condensed version is just as good. 


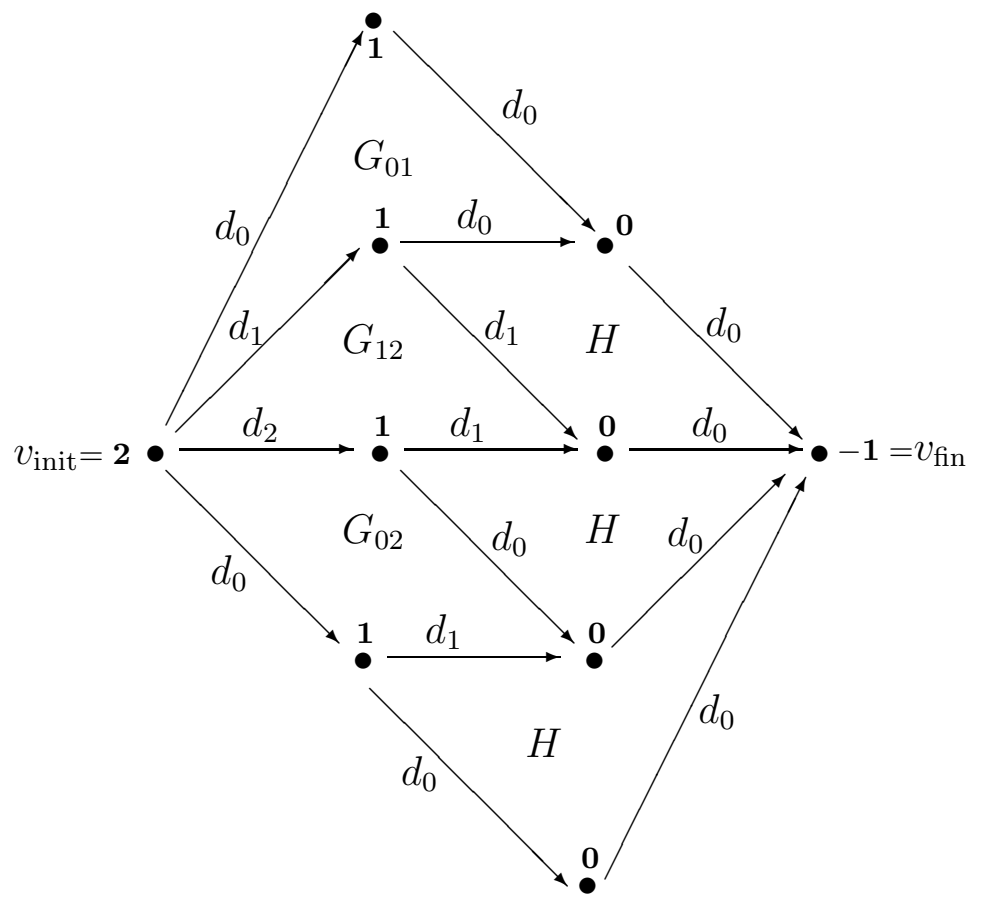

FIGURE 4.7. Lattice for 2-permutahedron

4.6. Example. For $n=2$ the lattice $\Gamma$ of $\S 4.3$ is described by Figure 4.7 .

We have not indicated any of the commuting triangles here, but only the commuting quadrilaterals which result directly from applying (4.4). Moreover, the true graph is not planar, since all edges with the same label and vertices should be identified, as well as the commuting quadrilaterals labeled $H$.

The minimal model for $W \Gamma$ is the 2-permutohedron $\mathrm{Pe}_{2}$ in Figure 4.8. Here each vertex or edge has been given two different labels - one corresponding to the description of $\Gamma$ in $\S 4.3$, and the other to the description of $\Gamma^{\prime}$ in $\$ 4.5$ (but we have abbreviated $(012,02, \emptyset)$, e.g., to $(012,02))$.

Similarly, for $n=3$ we obtain the 3-permutahedron $\mathrm{Pe}_{3}$ (with maximal chain $\left.d_{0} d_{1} d_{2} d_{3}\right)$ in Figure 4.9. Note that we have indicated only the labels of $\S 4.3$, where the chain $d_{i_{0}} d_{i_{1}} d_{i_{2}} d_{i_{3}}$, for example, is represented by $\left(i_{0}, i_{1}, i_{2}, i_{3}\right)$.

4.10. Remark. The higher homotopy operations associated to the family Perm were described explicitly in [B3, §5]. In fact, it was this case which motivated the search for a general definition of higher operations, which culminated in this paper.

4.11. Example. Let us describe explicitly the higher homotopy operation associated to the lattice $\Gamma$ of $\S 4.3$ for $n=2$.

A homotopy $\Gamma$-diagram is clearly given by maps $f_{0}, f_{1}, f_{2}: X \rightarrow Y, g_{0}, g_{1}: Y \rightarrow Z$, and $h: Z \rightarrow W$, as well as maps $A: Y \rightarrow W, B_{01}, B_{02}, B_{12}: X \rightarrow Z$ and $C: X \rightarrow W$, 


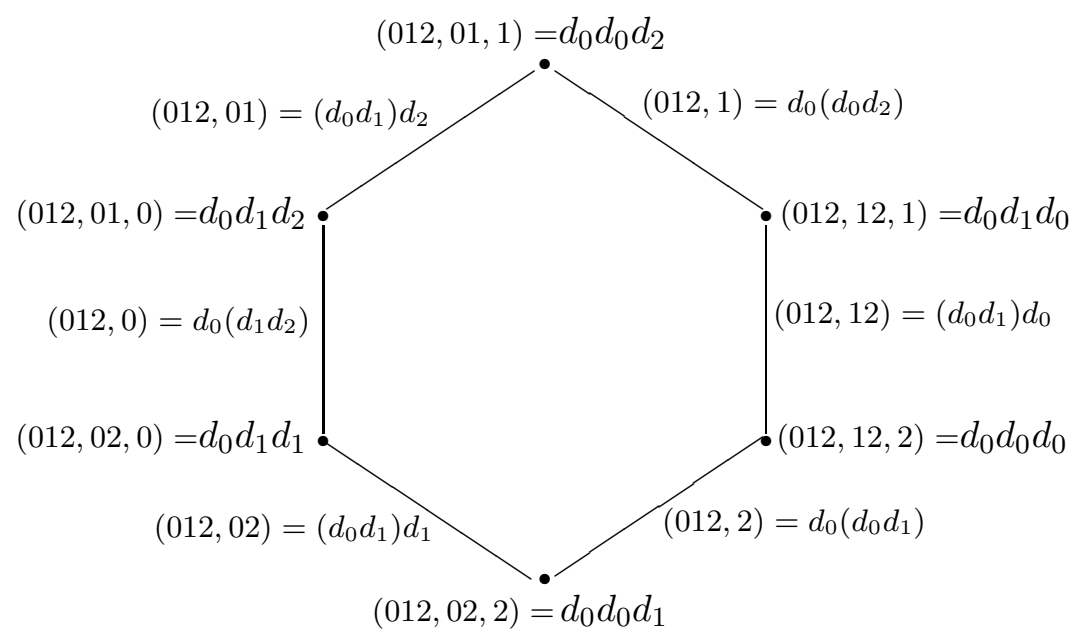

Figure 4.8. Two descriptions of $\mathrm{Pe}_{2}$

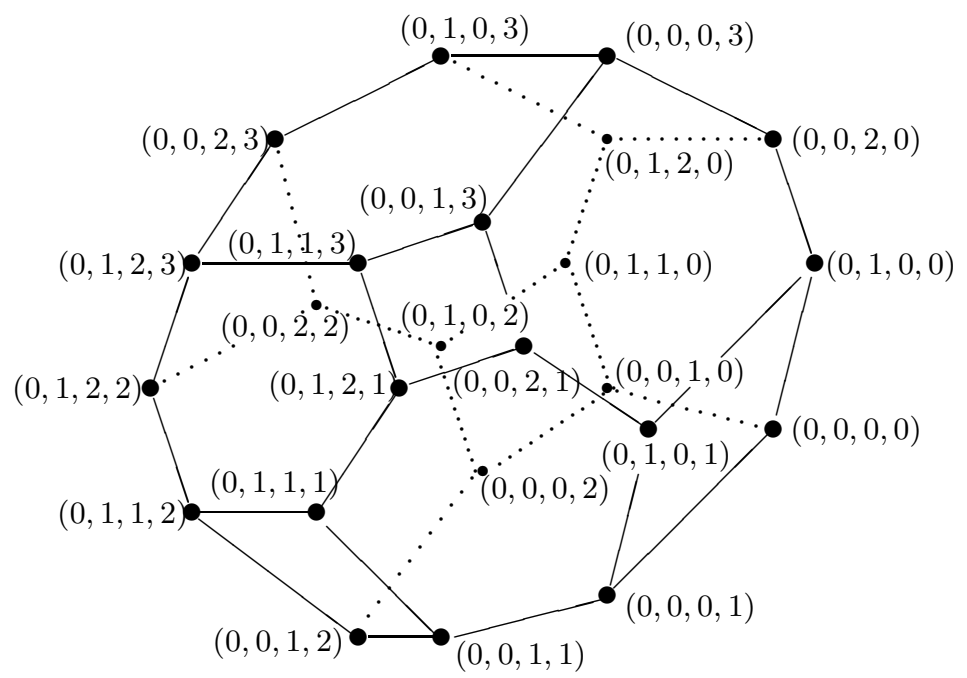

Figure 4.9. A depiction of $\mathrm{Pe}_{3}$

such that $\left[h g_{0}\right]=\left[h g_{1}\right]=[A], \quad\left[g_{0} f_{0}\right]=\left[g_{0} f_{1}\right]=\left[B_{01}\right], \quad\left[g_{1} f_{1}\right]=\left[g_{1} f_{2}\right]=\left[B_{12}\right]$, $\left[g_{0} f_{2}\right]=\left[g_{1} f_{1}\right]=\left[B_{02}\right]$ and $\left[h g_{0} f_{0}\right]=[C]$.

One readily verifies that the maps $A, \quad B_{01}, B_{02}, \quad B_{12}$ and $C$ are irrelevant to the homotopy operation, and may disregarded - this would correspond to taking the 'minimal model' of the hexagon $\mathrm{Pe}_{2}$ in Figure 4.8, instead of its decomposition into six cubes using the cubical structure of the $W$-construction.

Complete data are then given by explicit homotopies $H: h g_{0} \sim h g_{1}, G_{01}: g_{0} f_{0} \sim$ $g_{0} f_{1}, \quad G_{12}: g_{1} f_{1} \sim g_{1} f_{2}$ and $G_{02}: g_{0} f_{2} \sim g_{1} f_{1}$, which can be organized into a map $X \rtimes S^{1} \rightarrow W$.

The corresponding higher homotopy operation is then the subset $\left\langle h, g_{0}, g_{1}, f_{0}, f_{1}, f_{2}\right\rangle \subset$ $\left[X \rtimes S^{1}, W\right]$ formed by all complete data, as in Figure 4.12 (where we have also indicated 
by dotted lines the cubical decomposition provided by the maps $A, B_{01}, B_{02}$, and $\left.B_{12}\right)$.

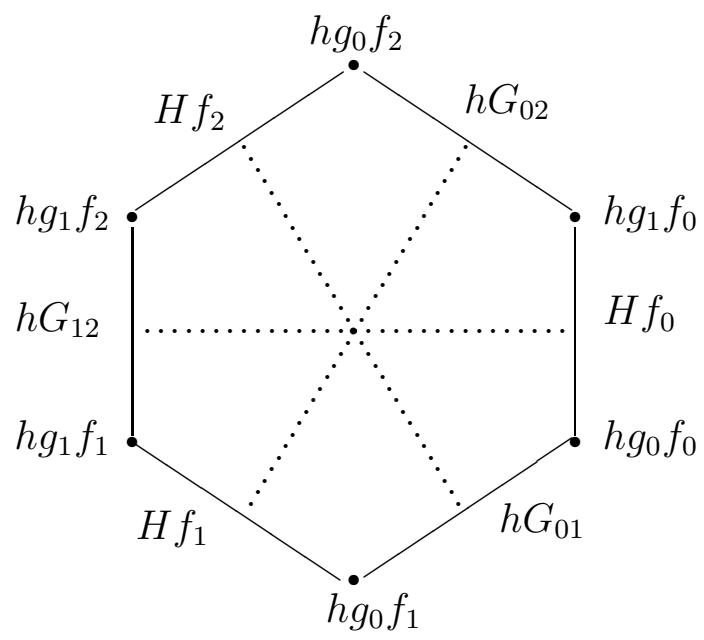

FIGURE 4.12. Complete data for the operation $\left\langle h, g_{0}, g_{1}, f_{0}, f_{1}, f_{2}\right\rangle$

To describe higher homotopy operations related to other polyhedra of this section is equally straightforward and we may safely leave these calculations to the reader as an exercise.

4.13. Simplices. Many other families of polytopes are obtained from Perm by considering relative higher homotopy operations (\$3.11), which has the effect of collapsing some of the cubes (or simplices) of $W \Gamma$.

Thus, the family $\boldsymbol{\Delta}=\{\Delta[n]\}_{n=0}^{\infty}$ of simplices arises from the same diagram $\mathbb{N}_{+}$as Perm, in the case where all the relations of (4.4) are assumed to hold on the nose, except for the single relation $\varepsilon d_{0}=\varepsilon d_{1}$. In this case the permutohedron $P e_{n}$ (corresponding to the subcategory $\Gamma$ of $\mathbb{N}_{+}^{\text {op }}$ with objects $\left.\{[-\mathbf{1}],[\mathbf{0}], \ldots,[\boldsymbol{n}]\}\right)$ collapses canonically to an $n$-simplex.

For example, Figure 4.14 shows the 3 -simplex corresponding to the possible decompositions of $\varepsilon d_{0} d_{1} d_{2}$.

4.15. Remark. The higher homotopy operations associated to the family $\Delta$ were described explicitly in [B4, §4].

4.16. Associahedra. Similarly, the family $\mathcal{A} s s=\left(K_{n}\right)_{n=0}^{\infty}$ of associahedra arises from a less drastic relative version of the diagram $\mathbb{N}_{+}$, using a procedure defined by Andy Tonks for collapsing certain faces of the permutohedron; this can be defined explicitly in terms of the lattice $\mathbb{N}_{+}$. We shall just give two examples, refering the reader to [To] for further details. 


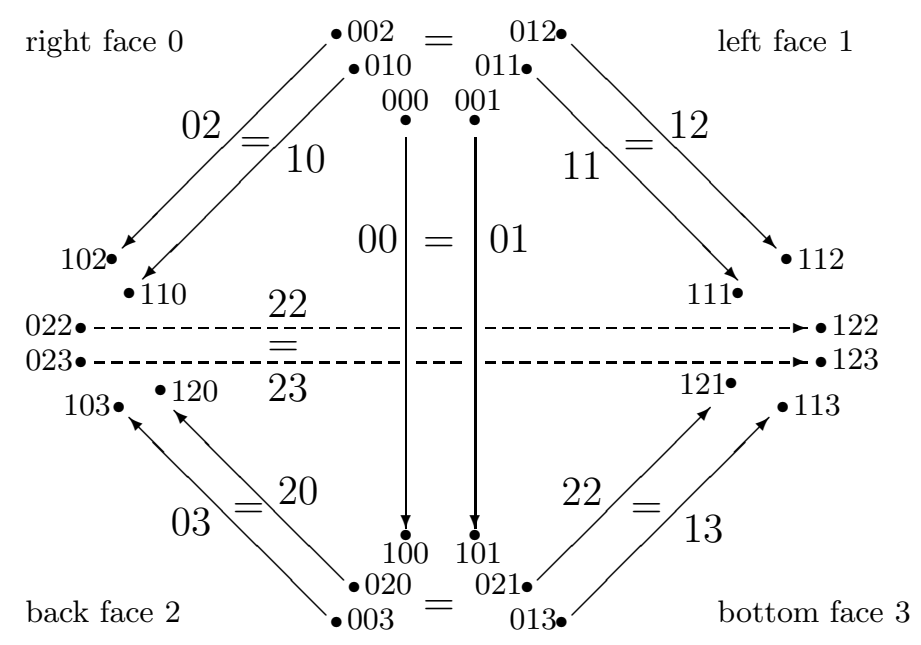

Figure 4.14. The 3-simplex

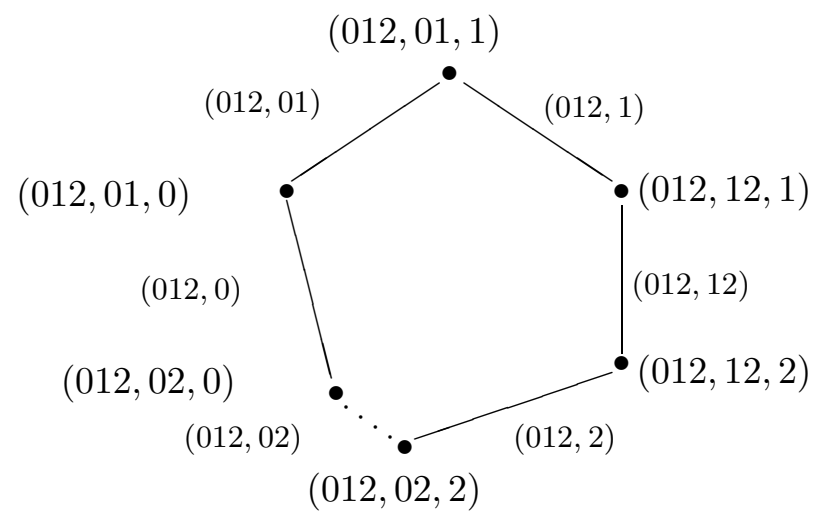

FiguRE 4.18. The 2-associahedron

4.17. Example. If we think of the 2-permutohedron as the face polyhedron for $\Delta[2]$ (\$4.5), then the edge $(012,02)$ in Figure 4.8 should be collapsed to yield the 2associahedron in Figure 4.18, where the dotted edge is collapsed.

Similarly, in the 3-permutahedron of Figure 4.9 with the labeling of $\$ 4.5$, we collapse each of the edges

$$
\begin{gathered}
(0123,012,02),(0123,023,02),(0123,023,03),(0123,013,03),(0123,023,2), \\
(0123,023,3),(0123,123,13),(0123,013,13),(0123,013,1) \text { and }(0123,013,0),
\end{gathered}
$$

of $\mathrm{Pe}_{3}$ to a point, so that each of the 2-dimensional faces

$$
(0123,13),(0123,013),(0123,02),(0123,03) \text { and }(0123,023)
$$

of $\mathrm{Pe}_{3}$ is collapsed to an edge in the resulting 3-associahedron.

One can also describe the $n$-associahedron directly in terms of bracketing on $(n+2)$ symbols (cf. [St1, §2]) - equivalently, in terms of labelled trees (cf. [St3, §11]) - or as a 
truncated $n$-simplex (cf. [SS, §5.1]); but these descriptions do not fit into our framework of lattices.

4.19. Remark. The higher homotopy operations associated to the family $\mathcal{A} s s$ have not been described in this language, but implicitly they motivated Stasheff's original definition in St1].

4.20. Example. Let $\Gamma$ be as in 4.3. Consider the relative case when all equations of (4.4) are strictly preserved except for

$$
d_{0}^{k-1} d_{1}^{k}=d_{0}^{k-1} d_{0}^{k}, 1 \leq k \leq n .
$$

The corresponding family of polyhedra is the sequence $\mathrm{C} u$ of cubes presented as quotients of the permutohedra, as illustrated for $n=2$ in Figure 4.21.

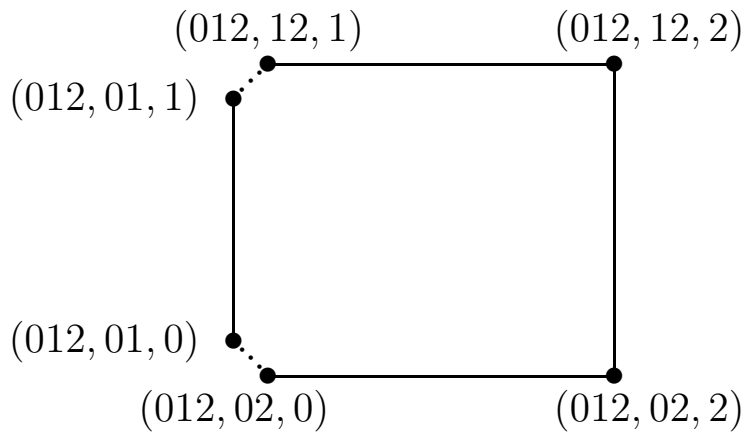

Figure 4.21. The 2-cube as a quotient of the 2-permutahedron

4.22. Example. Consider two copies $\Gamma^{\prime}$ and $\Gamma^{\prime \prime}$ of the lattice from 4.3, with objects $\left\{-\mathbf{1}^{\prime}, \mathbf{0}^{\prime}, \ldots, \mathbf{n}^{\prime}\right\}$ and $\left\{-\mathbf{1}^{\prime \prime}, \mathbf{0}^{\prime \prime}, \ldots, \mathbf{n}^{\prime \prime}\right\}$ respectively. Let $\Gamma$ be the lattice obtained from the disjoint union $\Gamma^{\prime} \sqcup \Gamma^{\prime \prime}$ by adding arrows $f_{i}: \mathbf{i}^{\prime} \rightarrow \mathbf{i}^{\prime \prime}$ for $-1 \leq i \leq n$ satisfying

$$
f_{k-1} d_{j}^{k}=d_{j}^{k} f_{k}, \text { for } 0 \leq k \leq n \text { and } 0 \leq j \leq k
$$

See Figure 4.24.

Obviously, a $\Gamma$-diagram consists of two truncated $\Delta$-simplicial spaces and their $\Delta$ simplicial homomorphism.

Consider the relative case when all simplicial identities are strictly satisfied and also all the identities of (4.23) are strict, except for the case when $j=k$. The relevant polyhedron is now the $(n+1)$-simplex obtained as a quotient of the permutahedron with $(n+2)$ !-vertices indexed by

$$
d_{i_{0}}^{0} d_{i_{1}}^{1} \cdots d_{i_{n}}^{n} f_{n}, d_{i_{0}}^{0} d_{i_{1}}^{1} \cdots d_{i_{n-1}}^{n-1} f_{n-1} d_{i_{n}}^{n}, \ldots d_{i_{0}}^{0} f_{0} d_{i_{1}}^{1} \cdots d_{i_{n}}^{n} \text { and } f_{-1} d_{i_{0}}^{0} d_{i_{1}}^{1} \cdots d_{i_{n}}^{n},
$$

for $0 \leq i_{j} \leq j, 0 \leq j \leq n$. This is illustrated, for $n=1$, in Figure 4.25 . 


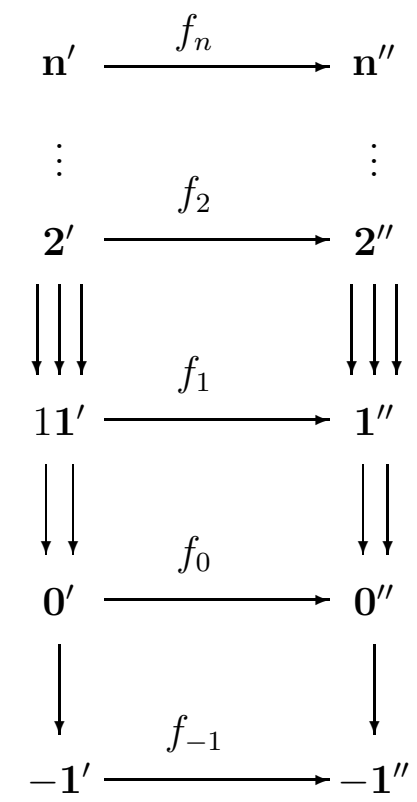

Figure 4.24. The category $\Gamma$

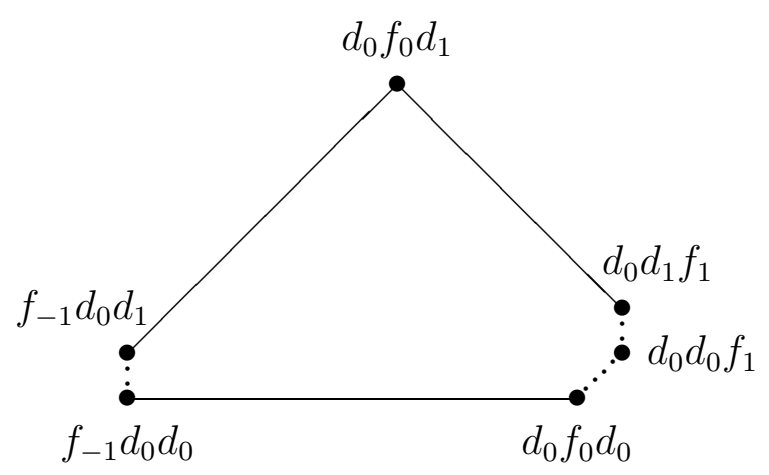

Figure 4.25. The 2-simplex as a quotient of the 2-permutahedron

\section{MASSEY PRODUCTS}

We now show how Massey products fit into our general framework.

5.1. Higher Whitehead products. Because it fits better with our original definition, we start with the Lie analogue, sometimes called higher-order Whitehead products:

Any three elements in $\pi_{*} X$ determine a map of the form $F: S^{r} \vee S^{s} \vee S^{t} \rightarrow X$; if two of their pairwise Whitehead products vanish, $F$ fits into the diagram in Figure 5.2, where $w_{r, s}: S^{r+s-1} \rightarrow S^{r} \vee S^{s}$ is the Whitehead product map (and the unmarked maps are the obvious inclusions or projections).

If we think of this as a functor $\mathcal{A}: \Gamma \rightarrow h o \mathcal{T}_{*}$ for the corresponding lattice $\Gamma$, and try to rectify it (relative to the specified null maps - or even relative to the subdiagram involving only maps between wedges of spheres), the first (and only) obstruction $\langle\langle\mathcal{A}\rangle\rangle \subseteq$ $\pi_{r+s+t-1} X$ is called a secondary Whitehead product. One has $n$-th order analgues defined 


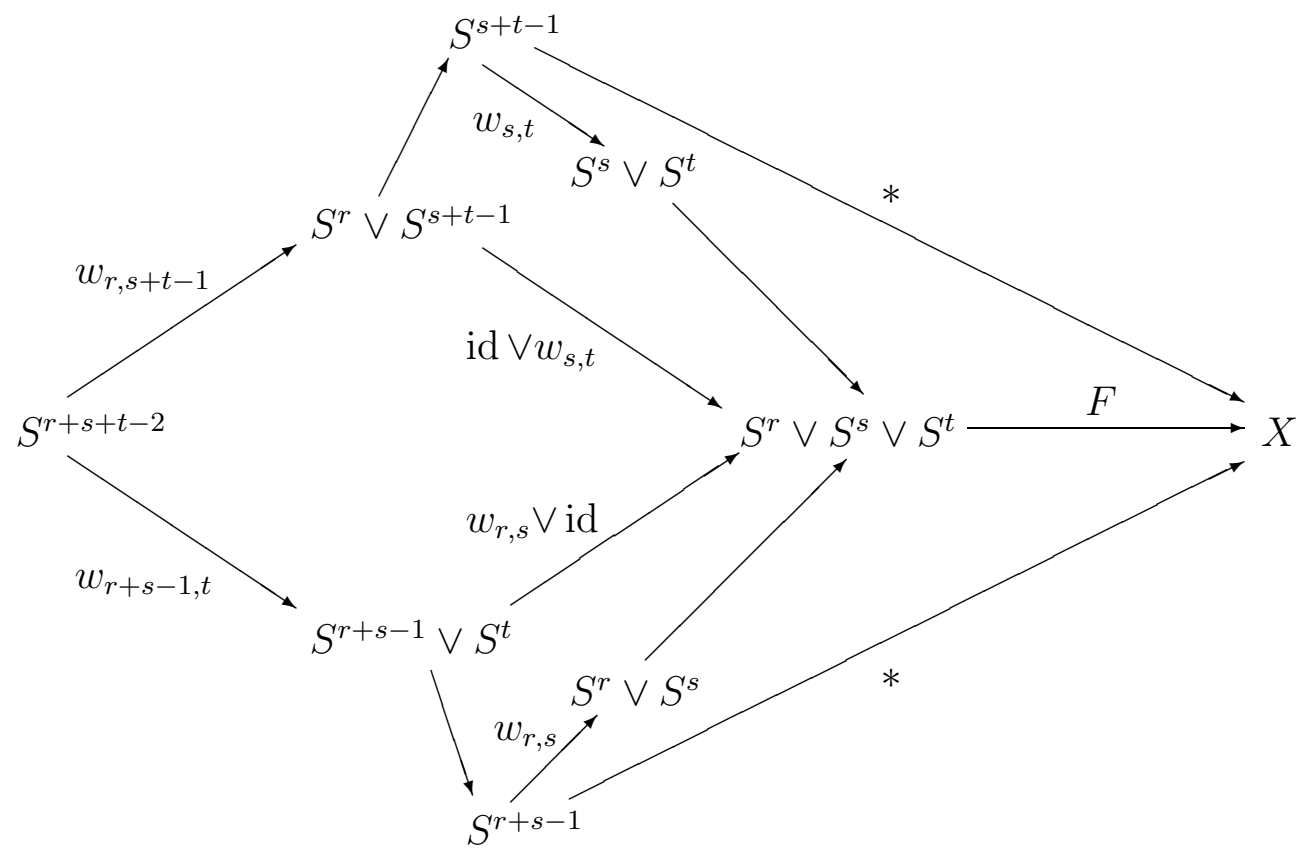

FiguRE 5.2. The diagram defining triple Whitehead products

for sets of $n+1$ elements in $\pi_{*} X$ for which all lower-order Whitehead products vanish coherently (see [P1]).

Of course, all this is valid not only in the category of topological spaces, but also in the category of differential graded Lie algebras (DGLs); if we consider connected DGLs over $\mathbb{Q}$, we obtain the usual Lie-Massey products of rational homotopy theory (cf. Ald, R] or [Ta, V.1]).

5.3. Generalized Massey products. If $K=\left(\underline{K}_{n}\right)_{n=0}^{\infty}$ is any associative ring spectrum, one can define the corresponding Massey products by dualizing diagram 4.25 above, replacing the Whitehead products $w_{r, s}: S^{r+s-1} \rightarrow S^{r} \vee S^{s}$ by the multiplication maps $m^{r, s}: \underline{K}_{r} \times \underline{K}_{s} \rightarrow \underline{K}_{r+s}$ :

However, in this case it is usual to dualize our description of the higher order homotopy operation associated to the corresponding homotopy-commutative diagram $\mathcal{A}: \Gamma \rightarrow \mathcal{T}_{*}$, and defining $\overline{\langle\langle\mathcal{A}\rangle\rangle}$ to be the collection of adjoints in $\left[X, \Omega \underline{K}_{r+s+t}\right]$ to the classes $\langle\langle\mathcal{A}\rangle\rangle \subseteq\left[\Sigma X, \underline{K}_{r+s+t}\right]$ (compare $\left.[\overline{\mathrm{P} 2}]\right)$.

When $K=H R$ is the Eilenberg-Mac Lane spectrum for the ring $R$, we obtain the usual Massey products in $H^{*}(X ; R)$ (see [M] and [MU, §2]), and their higher-order generalizations (cf. [K, Mi]). The $K$-theory and cobordism versions have been considered in [Sn and Al] respectively, (and elsewhere). Of course, one can define such products in any model category with products - e.g., for differential graded algebras. 


\section{REFERENCES}

[As] J.F. Adams, "On the non-existence of elements of Hopf invariant one", Ann. Math. (2) 72 (1960), No. 1, pp. 20-104.

[Am] J. Adem, "The iteration of the Steenrod squares in algebraic topology", Proc. Nat. Acad. Sci. USA38 (1952),pp. 720-726.

[Ald] C. Allday, "Rational Whitehead products and a spectral sequence of Quillen", Pac. J. Math. 46 (1973) No. 2, pp. 313-323.

[Ale] J.C. Alexander, "Cobordism Massey products", Trans. AMS 166 (1972), 197-214.

[BJM] M.G. Barratt, J.D.S. Jones \& M.E. Mahowald, "Relations amongst Toda brackets and the Kervaire invariant in dimension 64", J. Lond. Math. Soc. 30 (1984), pp. 533-550.

[Ba] H.J. Baues, "Geometry of loop spaces and the cobar construction", Mem. AMS 230, AMS, Providence, RI, 1980.

[B1] D. Blanc, "A Hurewicz spectral sequence for homology", Trans. AMS 318 (1990) No. 1, pp. 335-354.

[B2] D. Blanc, "Operations on resolutions and the reverse Adams spectral sequence", Trans. AMS 342 (1994) No. 1, pp. 197-213.

[B3] D. Blanc, "Higher homotopy operations and the realizability of homotopy groups", Proc. Lond. Math. Soc. (3) 70 (1995), pp. 214-240.

[B4] D. Blanc, "Homotopy operations and the obstructions to being an $H$-space", Manus. Math. 88 (1995) No. 4, pp. 497-515.

[Bo] J.M. Boardman, "Homotopy structures and the language of trees", In Algebraic Topology, Proc. Symp. Pure Math. 22, AMS, Providence, RI, 1971, pp. 37-58.

[BV] J.M. Boardman \& R.M. Vogt, Homotopy Invariant Algebraic Structures on Topological Spaces, Springer-Verlag Lec. Notes Math. 347, Berlin-New York, 1973.

[CP] J.-M. Cordier \& T. Porter, "Vogt's theorem on categories of homotopy coherent diagrams", Math. Proc. Camb. Phil. Soc. 100 (1986), No. 1, pp. 65-90.

[DKS] W.G. Dwyer, D.M. Kan, \& J.H. Smith, "Homotopy commutative diagrams and their realizations", J. Pure Appl. Alg., 57 (1989), No. 1, pp. 5-24.

$[\mathrm{H}] \quad$ D.N. Holtzman, "Higher order cohomology operations in the $p$-torsion-free category", Neder. Akad. Weten. Proc. 44 (1982), No. 2, pp. 183-200.

[Kl] S. Klaus, "Towers and Pyramids, I", Fund. Math 13 (2001), No. 5, pp. 663-683.

[KK] A. Kock \& L. Kristensen, "A secondary product stucture in cohomology theory", Math. Scand. 17 (1965), pp. 113-149.

[K] D.P. Kraines, "Massey higher products", Trans. AMS 124 (1966), 431-449.

[Kr] L. Kristensen, "On secondary cohomology operations", Math. Scand. 12 (1963), pp. 57-82.

[Mc] S. Mac Lane, Categories for the Working Mathematician, Springer-Verlag Grad. Texts in Math. 5, Berlin-New York, 1971.

[MP] M.E. Mahowald \& F.P. Peterson, "Secondary operations on the Thom class", Topology 2 (1964), pp. 367-377

[Ms] W.S. Massey, "A new cohomology invariant of topological spaces", Bull. AMS $5 \mathbf{7}$ (1951), p. 74 .

[MU] W.S. Massey \& H. Uehara, "The Jacobi identity for Whitehead products", in Algebraic geometry and topology, Princeton U. Press, Princeton, 1957, pp. 361-377.

[Mar] H.R. Margolis, Spectra and the Steenrod Algebra: Modules over the Steenrod Algebra and the Stable Homotopy Category, North-Holland, Amsterdam-New York, 1983.

[Mau] C.R.F. Maunder, "Cohomology operations of the $N$-th kind", Proc. Lond. Math. Soc. Ser. (2) 13 (1963), pp. 125-154.

[May] J.P. May, The Geometry of Iterated Loop Spaces, Springer-Verlag Lec. Notes Math. 271, Berlin-New York, 1972.

[Mi] R.J. Milgram, "Iterated loop spaces", Ann. Math. (2) 84 (1966), pp. 386-403.

[Mo] M. Mori, "On higher Toda brackets", Bull. College Sci. Univ. Ryukyus 35 (1983), pp. 1-4.

[PS] F.P. Peterson \& N. Stein, "Secondary cohomology operations: two formulas", Amer. J. Math. 81 (1959), pp. 231-305.

[P1] G.J. Porter, "Higher order Whitehead products", Topology 3 (1965), 123-165. 
[P2] G.J. Porter, "Higher products", Trans. AMS 148 (1970), 315-345.

[R] V.S. Retakh, "Lie-Massey brackets and $n$-homotopically multiplicative maps of differential graded Lie algebras", J. Pure \& Appl. Alg. 89 (1993) No. 1-2, pp. 217-229.

[Sc] P.-H. Schoute, "Analytic treatment of the polytopes regularly derived from the regular polytopes", Verh. Kon. Akad. Wet. Amst., 11, 1911.

[SV] R. Schwänzl \& R.M. Vogt, "Coherence in homotopy groups actions", in Transformation Groups, Poznan 1985, Lecture Notes in Mathematics 1217 (1986), pp. 363-390.

[Se] G.B. Segal, "Categories and cohomology theories", Topology 13 (1974), pp. 293-312.

[SS] S. Shnider \& S. Sternberg, Quantum groups: from coalgebras to Drinfel'd algebras, International Press Grad. Texts in Math. Phys. II, Cambridge, MA, 1993.

[Sn] V.P. Snaith, "Massey products in K-theory", Proc. Camb. Phil. Soc. 68 (1970), 303-320.

[Sp1] E.H. Spanier, "Secondary operations on mappings and cohomology", Ann. Math. (2) 75 (1962) No. 2, pp. 260-282.

[Sp2] E.H. Spanier, "Higher order operations", Trans. AMS 109 (1963), pp. 509-539.

[St1] J.D. Stasheff, "Homotopy associativity of $H$-spaces, I", Trans. AMS 108 (1963) pp. 275292.

[St2] J.D. Stasheff, "Homotopy associativity of $H$-spaces, II", Trans. AMS 108 (1963) pp. 293312 .

[St3] J.D. Stasheff, H-spaces from a Homotopy Point of View, Springer-Verlag Lec. Notes Math. 161, Berlin-New York, 1970.

[Ta] D. Tanré, Homotopie Rationelle: Modèles de Chen, Quillen, Sullivan, Springer-Verlag Lec. Notes Math. 1025, Berlin-New York, 1983.

[T1] H. Toda, "Generalized Whitehead products and homotopy groups of spheres", J. Inst. Polytech. Osaka City U., Ser. A, Math. 3 (1952), pp. 43-82.

[T2] H. Toda, Composition methods in the homotopy groups of spheres, Adv. in Math. Study 49, Princeton U. Press, Princeton, 1962.

[To] A.P. Tonks, "Relating the associahedron and the permutohedron", in J.L. Loday, J.D. Stasheff, \& A.A. Voronov, eds., Operads: Proceedings of Renaissance Conferences (Hartford, CT/Luminy, 1995 Contemp. Math. 202, AMS, Providence, RI 1997, pp. 33-36.

[V] R.M. Vogt, "Cofibrant operads and universal $E_{\infty}$ operads", in R.M. Vogt editor, Workshop on Operads, Osnabrück, June 1998, Preprint of Univ. Bielefeld 1999, pp. 81-89.

[Wa] G. Walker, "Long Toda brackets", in Proc. Adv. Studies Inst. on Algebraic Topology, vol. III, Aarhus U. Mat. Inst. Various Publ. Ser. 13, Aarhus 1970, pp. 612-631.

[Wh] G.W. Whitehead, Homotopy Theory, M.I.T. Press, Cambridge, MA, 1953.

Dept. of Mathematics, Univ. of Haifa, 31905 Haifa, IsRAel

Mathematical Inst. of the Academy, Žitná 25, 11567 Prague 1, Czech Republic

E-mail address: blanc@math.haifa.ac.il, markl@math.cas.cz 